\title{
General Variable Neighborhood Search for the Quote-Travelling Repairman Problem
}

\author{
Ha-Bang Ban \\ School of Information and Communication Technology \\ Hanoi University of Science and Technology, Hanoi, Vietnam
}

\begin{abstract}
The Quota-Travelling Repairman Problem (QTRP) tries to find a tour that minimizes the waiting time while the profit collected by a repairman is not less than a predefined value. The Q-TRP is an extended variant of the Travelling Repairman Problem (TRP). The problem is NP-hard problem; therefore, metaheuristic is a natural approach to provide near-optimal solutions for large instance sizes in a short time. Currently, several algorithms are proposed to solve the TRP. However, the quote constraint does not include, and these algorithms cannot be adapted to the Q-TRP. Therefore, developing an efficient algorithm for the Q-TRP is necessary. In this paper, we suggest a General Variable Neighborhood Search (GVNS) that combines with the perturbation and Adaptive Memory (AM) techniques to prevent the search from local optima. The algorithm is implemented with a benchmark dataset. The results demonstrate that good solutions, even the optimal solutions for the problem with 100 vertices, can be reached in a short time. Moreover, the algorithm is comparable with the other metaheuristic algorithms in accordance with the solution quality.
\end{abstract}

Keywords-Q-TRP; GVNS; AM; GRASP

\section{INTRODUCTION}

The Quota-Travelling Repairman Problem (Q-TRP) in the case of Travelling Repairman Problem (TRP) has been studied in the numerous articles [1], [2], [3], [4], [5], [6], [8], [10], [14], [15]. It is often called the Travelling Repairman Problem (TRP). The TRP takes a customer-oriented approach when the objective function is to minimize the total customers' waiting time. In addition, the TRP has many practical applications in, e.g., disk head scheduling [1], [2], [4], [5], [6]. In the general case, the Q-TRP is an extended version of the TRP case. Informally, the Q-TRP tries to find a tour that minimizes the waiting time while the profit collected by a repairman is not less than a predefined value of $P_{\text {min }}$. The practical application for the problem can be described as follows: A Repairman must sell a quota $P_{\min }$ of goods. Repairman knows the distances between cities and how many goods he could sell in his tour. His objective is to travel along a tour while minimizing the waiting time and selling the required quota of goods. We formulate the Q-TRP as followings: Let $K_{n}=(V, E)$ be a complete graph in which $V=\{1,2, \ldots n\}$, $E$ are a set of vertices and edges, respectively. For each edge $\left(v_{i}, v_{j}\right) \in E$, which connects the two vertices $v_{i}$ and $v_{j}$, there exist a cost $c\left(v_{i}, v_{j}\right)$ and the quantity of goods sold $r\left(v_{i}, v_{j}\right)$. Suppose that $T=\left(v_{1}, \ldots, v_{k}, \ldots, v_{n}\right)$ is a tour. Let $P\left(v_{1}, v_{k}\right)$ be the path from $v_{1}$ to $v_{k}$ on it and $l\left(P\left(v_{1}, v_{k}\right)\right)$ is its length. The waiting time of a vertex $v_{k}(1<k \leq n)$ on $T$ is calculated as follows:

$$
w\left(v_{k}\right)=\sum_{i=1}^{k-1} c\left(v_{i}, v_{i+1}\right) .
$$

The cost of the tour $T$ is calculated:

$$
L(T)=\sum_{k=1}^{n+1} w\left(v_{k}\right) .
$$

The quantity of goods sold on the tour is the sum of the amount of goods sold of its edges. The tour must satisfy the following constraint:

$$
\sum_{i=1}^{n} r\left(v_{i}, v_{i+1}\right) \geq P_{\min }
$$

The term $v_{i+1}$ for $i=n$ in the preceding formula coincides with $v_{1}$, so that the repairman in the very end returns to the vertex from which he started his trip. The solution then is a Hamiltonian Cycle.

Generally speaking, for NP-hard problems, some types of algorithms are applied to solve the Q-TRP. Firstly, exact algorithms find the optimal solution, but they can only solve the problem with small sizes. Secondly, an $\alpha$-approximation algorithm produces a solution with the cost of no more than $\alpha$ times the optimal solution. Lastly, metaheuristic performs well in practice, and their efficiency is evaluated on a benchmark dataset.

The algorithm is one of the first metaheuristics to solve this problem. In this paper, we propose a General Variable Neighborhood Search (GVNS) [13] that combines with the perturbation and Adaptive Memory (AM) techniques [11] to prevent the search from local optima. Currently, there is no metaheuristic to solve this problem can be found in the literature to compare directly. Therefore, it is difficult to evaluate the efficiency of the A-GVNS exactly. To overcome the issue, several state-of-the-art metaheuristic algorithms for the TRP is chosen to compare to the A-GVNS. The AGVNS is implemented with benchmark instances. The results demonstrate that good solutions, even the optimal solutions for the problem with 100 vertices, can be reached in a short time. Moreover, the algorithm is comparable with the other metaheuristic algorithms in accordance with the solution quality.

The rest of this paper is demonstrated as follows. Section 2 provides the algorithm. Section 3 presents computational evaluations. Sections 4 and 5 discuss and conclude, respectively.

\section{THE PROPOSED ALGORITHM}

The modified GVNS (notation: A-GVNS) includes the GRASP [7] in the construction phase, and then the GVNS [13], the Adaptive Memory [11], and perturbation technique 
[12] in the improvement phase, respectively. The good metaheuristic needs to maintain the balance between diversification and intensification. Diversification means to generate diverse solutions to explore the unvisited solution space, while intensification means to focus on the search in a current region by exploiting it. In the algorithm, the A-GVNS ensures the intensification while the techniques maintain the diversification. This combination maintains the simplicity spirit of the GVNS while it effectively explores the search space.

An outline of the A-GVNS is shown in Algorithm 3. The A-GVNS starts with iterations of the construction to create an initial solution. At each iteration in the A-GVNS algorithm, a random perturbation is picked to shake the solution, and the local search is used to improve it. The best solution obtained by using a local search is saved in the AM list. The best solution in the AM, in accordance with the formula (5), is used as a starting solution in the next iteration. The algorithm returns the best solution when its computation time reaches $t_{\max }$. We realize that the statements from line 16 to 20 are removed, the A-GVNS becomes the original GVNS.

\section{A. Neighborhood Structures}

Several neighborhoods are widely applied in the literature to explore the solution space of this problem [16]. Let us denote $N_{k}\left(k=1, \ldots, k_{\max }\right)$, a finite set of pre-selected neighborhood structures, and with $N_{k}(T)$ the set of solutions in the $k-t h$ neighborhood of $T$. We describe more details about seven neighborhoods as follows:

- forward $\left(N_{1}\right)$ pushes a vertex to move forward a position in the tour. Its complexity is $\Theta(n)$.

- backward $\left(N_{2}\right)$ pushes a vertex to move backward a position in the tour. Its complexity is $\Theta(n)$.

- $\quad$ shift $\left(N_{3}\right)$ locates a vertex to another position in the tour. Its complexity is $\Theta(n)$.

- adjacent-swap $\left(N_{4}\right)$ exchanges the positions of each pair of adjacent vertices in the tour. Its complexity is $\Theta(n)$.

- swap $\left(N_{5}\right)$ a pair of vertices in the tour are interchanged. Its complexity is $\Theta\left(n^{2}\right)$.

- 2-opt $\left(N_{6}\right)$ removes each pair of vertices from the tour, and the reconnects them according to the reversing order. Its complexity is $\Theta\left(n^{2}\right)$.

- Or-opt $\left(N_{7}\right)$ is located three adjacent vertices to another position of the tour. Its complexity is $\Theta\left(n^{2}\right)$.

In the Q-TRP, the calculation of a neighboring solutions' cost requires exactly $\Theta(n)$ time. It leads to $\Theta(n)$ operations for each move evaluation resulting in $\Theta\left(n^{3}\right)$ operations for a full neighborhood search.

\section{B. Penalty on Infeasible Solution}

The search is not constrained to feasible solutions. During the search, we penalize infeasible solutions by incorporating a penalty value. For each solution $T$, let $L(T)$ denote the total cost and let $V(T)$ denote the violation of the constraint of the quantity of goods sold. The total cost violation $V(T)$ is
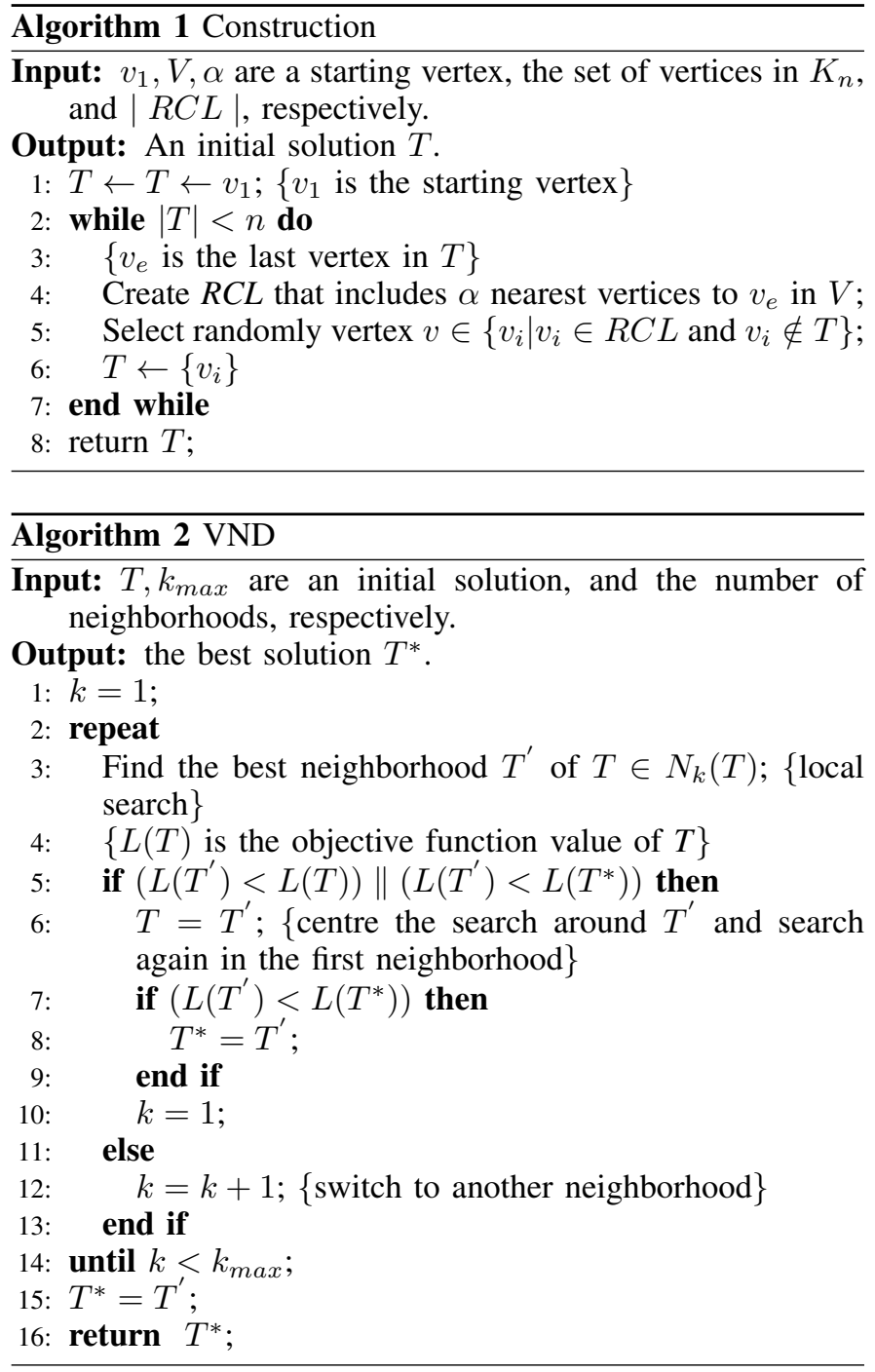

computed on a tour basis with respect to the value of $P_{\min }$. Specifically, it is equal to

$$
\max \left\{P_{\text {min }}-L P, 0\right\},
$$

where $L P$ is the quantity of goods sold in the current solution (feasible or infeasible).

Solutions are then evaluated according to the weighted fitness function $L^{\prime}=L+P F \times V(T)$, where $P F$ is the penalty value. Obviously, $L P \geq P_{\min }$ and $L^{\prime}=L$ when it is a feasible one.

\section{The Construction Phase}

Algorithm 1 shows the constructive procedure by using GRASP [7]. The algorithm works iteratively until an initial solution is found. At each step, we use a Restricted Candidate List (notation: $R C L$ ) of all non-visited vertices according to a greedy that evaluate the benefit of adding them in the solution. After that, one random vertex is chosen from the $R C L$ to add to the current partial tour. When all vertices are selected, it stops, and we obtain the initial solution. The size of $R C L$ controls the balance between greedy and random strategies. 


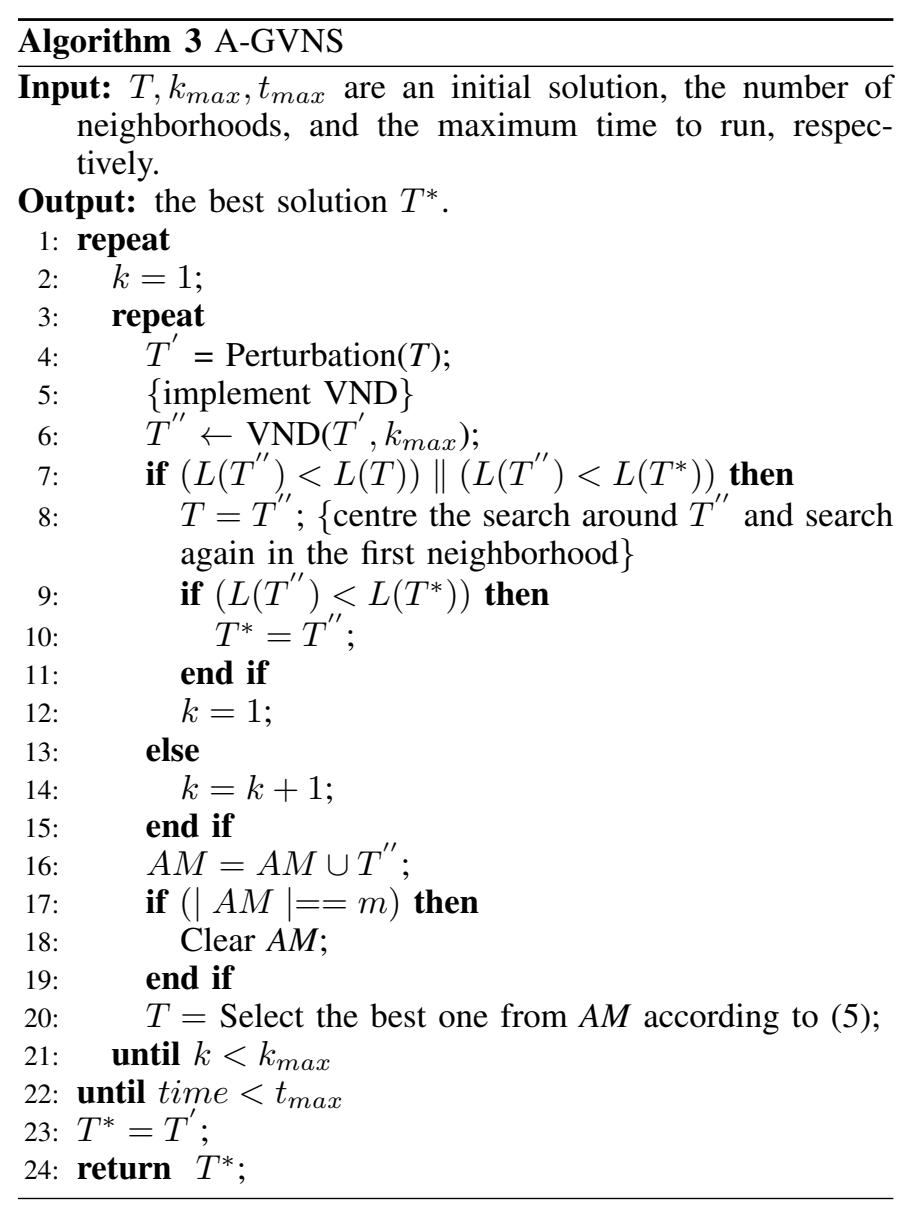

\section{The Improvement Phase}

The A-GVNS incorporates the Adaptive Memory [11] into the GVNS framework. The Adaptive Memory (AM) is a technique used in the local search provided by I. Mathlouthi et al. [11]. This technique not only allows to diversify the search by exploring solutions but also ensures to intensify the search to a promising region. However, when the technique does not provide enough diversification, the perturbation mechanism is applied. It drives the search to unexplored solution space. The combination helps the balance between diversification and intensification.

1) local search: Local search procedures are developed by combining the seven neighborhoods. From an initial solution, they generate their neighborhoods. The final solution should be a local minimum in comparison with all neighborhoods. The order of neighborhoods is deterministic. In a preliminary experiment, we realize that the results of the algorithm relatively depends on the order of exploring neighborhoods. The order of them is determined, from small to large size as follows: swap-adjacent, forward, backward, shift, swap, 2-opt, and oropt.

2) The adaptive memory: The Adaptive Memory (AM) [11] is a dynamic memory technique. It stores different solutions received by the local search improvement. For each solution in the AM, we count its cost and diversity in a set of solutions that present in the AM as follows:

$R(T)=\beta \times(|A M|-R F(T)+1)+(1-\beta) \times(|A M|-R D(T)+1)$,

where

$|A M|$ is the current size of $A M$.

$\beta \in[0,1]$. The parameter controls the balance between two rank values. $R F(T)$ is the rank of solution $T$ based on the objective function.

$R D(T)$ is the rank of solution $T$ based on its diversity contribution.

$$
\bar{d}(T)=\frac{\sum_{i=1}^{n} d\left(T, T_{i}\right)}{n}
$$

$d\left(T, T_{i}\right)$ is the metric distance between $T$, and $T_{i}$, and $\bar{d}(T)$ is the average distance metric of $T$ in the AM. In natural way, the distance is defined as the minimum number of transformations from $T$ to $T_{i}$, denoted $d\left(T, T_{i}\right)$. Since no polynomial method for computing $d\left(T, T_{i}\right)$ is known, $d\left(T, T_{i}\right)$ to be $n$ minus the number of vertices which has the same position in both of $T$ and $T_{i}$. The larger $\bar{d}(T)$ is, the higher its $R D(T)$ value is. The smaller $L(T)$ is, the higher its $R F(T)$ value is. The solution with the largest $R(T)$ value is selected from the $A M$.

3) The perturbation technique: Perturbation techniques prevent the algorithm from getting trapped into local optimal by driving the search to unexplored solution space. Two perturbations are selected as follows: 1) the double-bridge [12] is applied; 2) we select randomly two vertices and then swap them.

\section{EVALUATIONS}

We evaluate the A-GVNS in percent as followings: $\operatorname{Gap}_{1}[\%]=\frac{\text { best.sol-LB }}{L B} \times 100 \%$, and Gap $_{1}[\%]=$ $\frac{\text { best.sol-UB }}{U B} \times 100 \%$, where the $O P T, L B$, best.sol, and $U B$ correspond to the optimal solution, lower bound of the optimal solution, best, and the result of the VND, respectively. Note that: The value of $L B$ in the Q-TRP is the optimal solution of the TRP (the Q-TRP reduces to the TRP by removing the quantity of goods sold constraint). In the TRP, we can obtain the optimal solutions from the exact algorithm in [2]. However, the exact algorithm can solve the problem with up to 40 vertices. Therefore, for the small instances, the efficiency of the algorithms is evaluated according to the $G a p_{1}$ value.

Currently, we do not find any metaheuristic for this problem to compare. Therefore, our solution is compared with the result of the VND. In practice, the VND produces a quite good solution. Moreover, to present the efficiency of the AGVNS, we implement it on some TRP-instances. The results can compare directly with several state-of-the-art algorithms in [14], [15].

\section{A. Datasets}

The A-GVNS is implemented on CPU Core i7, 2.10 $\mathrm{GHz}$, and Ram $8 \mathrm{~GB}$. The parameters in the algorithm are determined through pilot experiments: $t_{\max }=1 \mathrm{~h},|A M|=$ $100, \beta=0.75, P F=10$, and $R C L=10$. In all tables, we report on the time since the best solution is reached.

The algorithms (VND, GVNS, and A-GVNS) are implemented on the benchmark for the Q-TRP and TRP [14], [17], [18]. These are: 1) A set of the Q-TRP instances is 
generated randomly as follows: The matrix costs, $c_{i j}$, are chosen randomly from integers in the range [0, 200]. The quantity of goods sold, $p_{i j}$, are chosen randomly from integers in the range $[0,200]$. The matrices are symmetric that satisfy the triangle inequality. Minimum quantity of goods sold, $P_{\min }$, is created using the following formula:

$$
P_{\text {min }}=\rho \times \sum_{i \in V} \sum_{j \in V} p_{i j} x_{i j}^{p}
$$

The values $x_{i j}^{p}$ represent the optimal solution of the problem

$$
\sum_{i=1}^{n} r\left(v_{i}, v_{i+1}\right) \rightarrow \max ,
$$

where the cost matrix is defined by the matrix $p_{i j}$. The problem with the above objective function has known as the MaxTSP. For the Max-TSP, we choose the Concorde tool [19] to solve exactly the problem. However, the tool solves the TSP problem with a minimization objective function. Since we are interested in the maximization problem, say $\max f$, then an equivalent minimization problem is $\min -f$. That is, minimizing $-f$ is the same as maximizing $f$. Therefore, we can adapt the tool to find $P_{\min }$. However, for the instances with 100 vertices, we have customized the way to compute $P_{\min }$. In the formula (7), the approximate solutions are used instead of the optimal solutions. Approximate solutions are computed using the Chained Lin-Kernighan algorithm in Concorde's tool. In the equation, $\rho$ is a parameter to control the tightness of the number of goods sold constraint. In the case of $\rho=0$, the optimal solution is not affected by the constraint. On the other hand, since $\rho=1$, it becomes very tight. The algorithm is implemented with the values of $\rho=0,0.5,0.75,0.95$, and 1. The size of the instances is selected between 30 to 100 vertices. The combination creates four hundred cases. The test instances are available at the web page [18]; 2) A. Salehipour et al. [14]: They provided five sets, where each of them is included 20 instances from 10 to 200 vertices, respectively; 3) Finally, several instances from the TSPLIB are selected by Abeledo et al. [1], [17].

Note that: The VND, GVNS, and A-GVNS are the algorithms in this article while GRASP-VNS, and ILS are the algorithms of Salihepour et al. 's [14], N. Silva et al. 's [15], respectively. Note that: The GVNS is the same as the AGVNS when statement lines from 16 to 20 in Algorithm 1 are removed.

\section{B. Results}

In the tables, aversol, best.sol, and $T$ are corresponding to the average, best solution, and average time in seconds of 10 executions, respectively, while cTime corresponds to the running time on a Pentium $4,2 \mathrm{GHz}$ according to the factors of Dongarra in [9]. Tables 1 to 20 show the results of the VND, GVNS, and A-GVNS, while in Tables 22 to 23, our results are compared with the previous algorithms in the TRP case. The average $\mathrm{Gap}_{2}$ calculated from Table 1 to 20, is illustrated in Table 21 .

In Tables 1 to 20, the VND, and GVNS obtain the feasible ones in some cases when the constraint is not tight. Since the constraint is very tight (the $\rho$ value is 1 ), no feasible solution can be reached by the VND, while several feasible ones can be found by the GVNS. On the other hand, the A-GVNS reaches the feasible solutions in 96 out of 100 tested instances. It is understandable because the larger and larger value of $\rho$ is the tighter and tighter the problem becomes. In Table 21, with the $\rho$ value of $0,0.5,0.75$, and 0.95 , the average $G_{a p}$ is from $2.43 \%$ to $25.50 \%$. It is implied that the solutions of the GVNS and A-GVNS are much better than those of the VND.

In Tables 1 to 10 show that in average $\operatorname{Gap}_{1}$, the solutions found by the GVNS, and A-GVNS are near to the optimal solutions since the difference between our solution and the lower bound is below $5.63 \%$. When the constraint is very tight, the solutions are within $10.02 \%$ of the optimum. Moreover, with $\rho=0$ (the Q-TRP becomes the TRP), the A-GVNS always finds the optimal solutions for the problem with up to 40 vertices. For the instances from 50 to 100 vertices, the exact algorithm in [2] cannot find the optimal solutions for the TRP. Therefore, the lower bounds are not remembered, and there are not the average $G a p_{1}$ values in these cases.

The GVNS and A-GVNS obtain better solutions than the VND. It is understandable since VND only ensures the intensification. Conversely, the A-GVNS maintains the diversification better by using the perturbation technique. Therefore, the perturbation technique plays an important role in improving the quality of the solution. In all results, the A-GVNS reaches better solutions than the GVNS does. Even in many cases with the value of $\rho$ of 1 , the A-GVNS finds the feasible solutions while the GVNS cannot do. Obviously, the AM technique brings efficiency to the A-GVNS when it can balance the intensification and diversification. More specifically, this technique allows implementing diversification in the search by exploring solutions that ensure the difference from each other. In addition, it intensifies the search to find better local optima in a promising solution space.

The experimental results show that the best-known TRPsolutions (the Q-TRP with $\rho=0$ ) can be infeasible solutions for the Q-TRP. Specifically, the best solutions of the TRP in Table 5, 10, 15, and 20 are not feasible for the Q-TRP when $\rho=1$. Therefore, the good methods for the TRP may not be applied to solve the Q-TRP. Developing an efficient algorithm for the Q-TRP is necessary.

Due to the lack of the works related to the metaheuristic algorithms of the Q-TRP, therefore we compare our solution with the algorithms for the TRP in Tables 22 and 23 [14], [15]. Specifically, in Table 22, our algorithm obtains better solutions than the GRASP-VNS [14] in all cases. In comparison with ILS [15], our solutions are the same for most of the instances. The results are significant because the algorithms in [14], [15] are the state-of-the-art metaheuristic algorithms. Moreover, our algorithm also is implemented on some TSP-instances. The optimal solutions for these instances can be extracted in [1]. The experimental results show that the optimal solutions can be reached for the problems with up to 100 vertices in several seconds [1] in Table 23. Obviously, our algorithm can solve well to the TRP.

The average running time of our algorithm is faster than the GRASP-VNS [14] and comparable with the ILS [15]. 


\section{Discussions}

Due to the NP-Hard problem, metaheuristic is a suitable approach for the Q-TRP. The metaheuristic can provide the near-optimal solution faster but without a guarantee of optimality.

Currently, several metaheuristic algorithms [1], [14], [15] are proposed to solve the TRP. However, the quote constraint in the works does not include, and their corresponding algorithms cannot be adapted to the Q-TRP. That means that we cannot use the above algorithms to solve the Q-TRP. Therefore, developing an efficient algorithm for the Q-TRP is necessary. There are no previous works in the literature to solve the Q-TRP, neither exact nor heuristically. Our contribution to this article is to propose the efficient algorithms for the problem. These algorithms are the first metaheuristics for the problem. In this work, three algorithms are used to solve the problem, such as the VND, GVNS, and GVNS-AM. Among the algorithms, the VND outputs the worse results. It is understandable because the VND only implements the intensification while the others maintain the diversification by using the perturbation technique. The GVNS-AM outperforms than the GVNS. Obviously, the AM brings the efficiency well since it balances between the diversity and intensification. The GVNS-AM is the most effective algorithm in terms of the quality of solution for the Q-TRP as well as TRP, although it consumes than the others. In the TRP case, it can find the optimal solutions to the problems with up to 100 vertices as well as provide the near-optimal solutions to the larger instances. Though our aim is not to propose metaheuristic for the TRP, the good results for this problem demonstrate the efficiency and broad applicability of our metaheuristics.

\section{CONCLUSIONS}

In this paper, we propose metaheuristic algorithms for the Q-TRP problem. The experimental results show that our algorithm obtains good solutions for the Q-TRP in a short time. In the TRP case, the optimal solutions can be reached for the instances with 100 vertices in some seconds. Our solutions are compared with the previous algorithms in both of the solution quality as well as running time.

\section{ACKNOWLEDGMENT} tion.

This research was supported by the Asahi Glass Founda-

\section{REFERENCES}

[1] Abeledo H.G., Fukasawa G., R. Pessoa R., and Uchoa A., 2010, The Time dependent Traveling Salesman Problem: Polyhedra and Branchcut-and Price Algorithm, Proc. SEA, pp. 202-213.

[2] Ban H.B., Nguyen K., Ngo M.C., and Nguyen D.N., 2013, An Efficient Exact Algorithm for Minimum Latency Problem, Jounal. PI, ; 10: 1-8.

[3] Bianco L., Mingozzi A., Ricciardelli S., 1993, The Travelling Salesman Problem with Cumulative Costs, Networks 23(2), pp. 81-91.

[4] Lucena A., 1990, Time-dependent Traveling Salesman Problem-the Repairman case, Journal. Networks; 20 (6), pp. 753-763.

[5] Blum A., Chalasani P., Coppersmith D., Pulleyblank W., Raghavan P., and Sudan M., 1994, The Minimum Latency Problem, Proc. STOC, pp. 163-171.

[6] Chaudhuri K., Goldfrey B., Rao S., and Talwar K., 2003, Path, Tree and Minimum Latency Tours, Proc. FOCS, pp. 36-45.

[7] Feo T.A., and Resende M.G.C., 1995, Greedy Randomized Adaptive Search Procedures, Journal. Global Opt., 6(2), pp. 109-133.

[8] Fischetti M., Laporte G., Martello S., 1993, The Delivery Man Problem and Cumulative Matroids. Oper Res, 41(6), pp. 1055-1064.

[9] Dongarra J. J., 2013, Performance of Various Computers Using Standard Linear Equations Software, Linpack Benchmark Report, University of Tennessee Computer Science Technical Report, CS-89-85.

[10] García A., Jodrá P., Tejel J., 2002, A note on the Traveling Repairman Problem. Networks 40(1), pp. 27-31.

[11] Mathlouthi I., Gendreau M., Potvin J. Y., 2018, A Metaheuristic Based on Tabu Search for Solving a Technician Routing and Scheduling Problem.

[12] Martin O., Otto S. W., Felten E. W., 1991, Large-step Markov chains for the Traveling Salesman Problem, Journal. Complex Systems, 5 (3), pp. 299-326.

[13] Mladenovic N., Hansen P., 1997, Variable Neighborhood Search, Journal. Operations Research, 24 (11), pp. 1097-1100.

[14] Salehipour A., Sorensen K., Goos P., and Braysy O., 2011, Efficient GRASP+VND and GRASP+VNS metaheuristics for the Traveling Repairman Problem, Journal. OR, 9 (2), pp. 189-209.

[15] Silva M., Subramanian A., Vidal T., Ochi L., 2012, A Simple and Effective Metaheuristic for the Minimum Latency Problem, Journal. EOR, 221(3), pp. 513-520.

[16] Aarts E.,Lenstra JK., 1997, Local Search in Combinatorial Optimization.New Jersey: Princeton University Press.

[17] http://elib.zib.de/pub/mp-testdata/tsp/tsplib/tsplib.html.

[18] https://sites.google.com/a/soict.hust.edu.vn/qtrp/home.

[19] http://www.math.princeton.edu/tsp/concorde.html. 
TABLE I. THE EXPERIMENTAL RESULTS FOR TEST-30-X WITH $\rho=1.0$

\begin{tabular}{|c|c|c|c|c|c|c|c|c|c|c|c|c|}
\hline \multirow{2}{*}{ instances } & \multirow{2}{*}{$L B$} & \multicolumn{3}{|c|}{ VND } & \multicolumn{4}{|c|}{ GVNS } & \multicolumn{4}{|c|}{ A-GVNS } \\
\hline & & best.sol & aver.sol & time & best.sol & aver.sol & Gap $_{1}$ & time & best.sol & aver.sol & Gap $_{1}$ & Time \\
\hline test-30-1 & 6415 & - & - & - & 6415 & 6415 & 0.00 & 1.88 & 6415 & 6415 & 0.00 & 1.89 \\
\hline test-30-2 & 7186 & - & - & - & 7263 & 7263 & 1.07 & 1.82 & 7263 & 7263 & 1.07 & 2.07 \\
\hline test-30-3 & 6286 & - & - & - & 6961 & 6961 & 10.74 & 1.85 & 6961 & 6961 & 10.74 & 2.14 \\
\hline test-30-4 & 6348 & - & - & - & 7093 & 7093 & 11.74 & 1.83 & 7093 & 7093 & 11.74 & 1.94 \\
\hline test-30-5 & 6239 & - & - & - & 7416 & 7416 & 18.87 & 1.86 & 7416 & 7416 & 18.87 & 2.11 \\
\hline test-30-6 & 6612 & - & - & - & 7004 & 7004 & 5.93 & 1.89 & 7004 & 7004 & 5.93 & 2.07 \\
\hline test-30-7 & 7305 & - & - & - & 7601 & 7601 & 4.05 & 1.91 & 7601 & 7601 & 4.05 & 1.80 \\
\hline test-30-8 & 5907 & - & - & - & 6283 & 6283 & 6.37 & 1.89 & 6283 & 6283 & 6.37 & 2.04 \\
\hline test-30-9 & 5988 & - & - & - & 6379 & 6379 & 6.53 & 1.98 & 6379 & 6379 & 6.53 & 1.95 \\
\hline test-30-10 & 7154 & - & - & - & 7366 & 7366 & 2.96 & 1.90 & 7366 & 7366 & 2.96 & 2.17 \\
\hline test-30-11 & 7154 & - & - & - & 7366 & 7366 & 2.96 & 1.99 & 7366 & 7366 & 2.96 & 1.80 \\
\hline test-30-12 & 6173 & - & - & - & 6655 & 6655 & 7.81 & 1.93 & 6655 & 6655 & 7.81 & 1.98 \\
\hline test-30-13 & 6031 & - & - & - & 6115 & 6115 & 1.39 & 1.99 & 6115 & 6115 & 1.39 & 1.97 \\
\hline test-30-14 & 5852 & - & - & - & 6250 & 6250 & 6.80 & 1.85 & 6250 & 6250 & 6.80 & 1.98 \\
\hline test-30-15 & 6059 & - & - & - & 6457 & 6457 & 6.57 & 1.94 & 6457 & 6457 & 6.57 & 2.11 \\
\hline test-30-16 & 6669 & - & - & - & 6884 & 6884 & 3.22 & 1.86 & 6884 & 6884 & 3.22 & 1.93 \\
\hline test-30-17 & 6937 & - & - & - & 7302 & 7302 & 5.26 & 1.93 & 7302 & 7302 & 5.26 & 2.11 \\
\hline test-30-18 & 6862 & - & - & - & 6967 & 6967 & 1.53 & 1.94 & 6967 & 6967 & 1.53 & 1.99 \\
\hline test-30-19 & 7469 & - & - & - & 7558 & 7558 & 1.19 & 1.81 & 7558 & 7558 & 1.19 & 1.81 \\
\hline test-30-20 & 6240 & - & - & - & 6508 & 6508 & 4.29 & 1.85 & 6508 & 6508 & 4.29 & 1.87 \\
\hline Aver & & & & & & & 5.46 & & & & 1.99 & \\
\hline
\end{tabular}

$L B$ : Since $\rho=0$, the Q-TRP becomes to the TRP. The optimal solution of the TRP found by using the exact algorithm in [2] are the lower bound of the optimal solution of the Q-TRP.

TABLE II. The EXPERIMENTAL ReSUlts FOR TEST-30-X With $\rho=0.95$

\begin{tabular}{|c|c|c|c|c|c|c|c|c|c|c|c|c|c|c|c|}
\hline \multirow{2}{*}{ instances } & \multirow{2}{*}{$L B$} & \multicolumn{4}{|c|}{ VND } & \multicolumn{5}{|c|}{ GVNS } & \multicolumn{5}{|c|}{ A-GVNS } \\
\hline & & best.sol & aver.sol & Gap $_{1}$ & time & best.sol & aver.sol & Gap $_{1}$ & $\mathrm{Gap}_{2}$ & time & best.sol & aver.sol & Gap $_{1}$ & Gap $_{2}$ & time \\
\hline test-30-1 & 6415 & 6914 & 7230.27 & 7.78 & 0.36 & 6719 & 7249.73 & 4.74 & -2.82 & 1.75 & 6675 & 7108.27 & 4.05 & -3.46 & 1.87 \\
\hline test-30-2 & 186 & 7807 & 8203.91 & 8.64 & 0.16 & 7402 & 7557.18 & 3.01 & -5.19 & 1.7 & 7384 & 7488.45 & 2.76 & -5.42 & 1.84 \\
\hline test-30-3 & 286 & 7343 & 7826.64 & 16.82 & 0.3 & 6404 & 6570.18 & 1.88 & -12.79 & 1.72 & 6347 & 6508.36 & 0.97 & -13.56 & 2.07 \\
\hline test-30-4 & 348 & 6645 & 7020.36 & 4.68 & 0.17 & 6616 & 6763.36 & 4.22 & -0.44 & 1.74 & 6567 & 3.91 & 3.45 & -1.17 & 1.85 \\
\hline test-30-5 & 239 & 6656 & 7078.27 & 6.68 & 0.13 & 6489 & 6660.73 & 4.01 & -2.51 & 1.81 & 6288 & 6585.27 & 0.79 & -5.53 & 1.8 \\
\hline test-30-6 & 612 & 7287 & 7533.64 & 10.21 & 0.27 & 7122 & 7233.36 & 7.71 & -2.26 & 1.74 & 7113 & 7190.18 & 7.58 & -2.39 & 1.81 \\
\hline test-30-7 & 305 & 7658 & 7756.36 & 4.83 & 0.23 & 7598 & 7827.64 & 4.01 & -0.78 & 1.8 & 7367 & 7723.64 & 0.85 & -3.80 & 1.92 \\
\hline test-30-8 & 5907 & 6479 & 6676.64 & 9.68 & 0.23 & 6222 & 6298.45 & 5.33 & -3.97 & 1.74 & 6037 & 6254.36 & 2.20 & -6.82 & 1.83 \\
\hline test-30-9 & 5988 & 6555 & 6756.36 & 9.47 & 0.29 & 6454 & 6593.64 & 7.78 & -1.54 & 1.82 & 6408 & 6530.73 & 7.01 & -2.24 & 1.87 \\
\hline test-30-10 & 7154 & 7899 & 8265.18 & 10.41 & 0.32 & 7563 & 7937.91 & 5.72 & -4.25 & 1.8 & 7333 & 7767.09 & 2.50 & -7.17 & 1.95 \\
\hline test-30-11 & 7154 & 7899 & 8265.18 & 10.41 & 0.2 & 7563 & 7937.91 & 5.72 & -4.25 & 1.78 & 7333 & 7767.09 & 2.50 & -7.17 & 1.89 \\
\hline test-30-12 & 6173 & 7395 & 7532.64 & 19.80 & 0.29 & 6355 & 6576.09 & 2.95 & -14.06 & 1.75 & 6336 & 6501.73 & 2.64 & -14.32 & 1.83 \\
\hline test-30-13 & 6031 & 6962 & 7323.64 & 15.44 & 0.22 & 6556 & .18 & 8.71 & -5.83 & 1.74 & 6302 & .27 & 4.49 & -9.48 & 1.95 \\
\hline test-30-14 & 5852 & 6785 & 7538.82 & 15.94 & 0.34 & 6234 & 6467.27 & 6.53 & -8.12 & 1.79 & 6126 & 6379.73 & 4.68 & -9.71 & 1.83 \\
\hline test-30-15 & 6059 & 6571 & 6698.45 & 8.45 & 0.33 & 6174 & 6405.18 & 1.90 & -6.04 & 1.72 & 6158 & 6333.73 & 1.63 & -6.29 & 1.88 \\
\hline test-30-16 & 6669 & 7226 & 7403.45 & 8.35 & 0.17 & 6794 & 6920.45 & 1.87 & -5.98 & 1.75 & 6777 & 6863.09 & 1.62 & -6.21 & 1.96 \\
\hline test-30-17 & 6937 & 7644 & 7782.45 & 10.19 & 0.27 & 7142 & 7314.82 & 2.96 & -6.57 & 1.79 & 7063 & 7244.18 & 1.82 & -7.60 & 1.84 \\
\hline test-30-18 & 6862 & 7527 & 7650.18 & 9.69 & 0.26 & 6996 & 7329 & 1.95 & -7.05 & 1.81 & 6977 & 7179.73 & 1.68 & -7.31 & 2.07 \\
\hline test-30-19 & 7469 & 8108 & 8259.27 & 8.56 & 0.25 & 7887 & 8062.82 & 5.60 & -2.73 & 1.73 & 7858 & 8000.73 & 5.21 & -3.08 & 1.9 \\
\hline test-30-20 & 6240 & 6841 & 7064.55 & 9.63 & 0.34 & 6566 & 6666.73 & 5.22 & -4.02 & 1.86 & 6506 & 6621.45 & 4.26 & -4.90 & 1.8 \\
\hline Aver & & & & 10.28 & 0.26 & & & 4.59 & -5.06 & 1.77 & & & 3.13 & -6.38 & 1.89 \\
\hline
\end{tabular}


TABLE III. The EXPERIMENTAL RESUlts FOR TEST-30-X With $\rho=0.75$

\begin{tabular}{|c|c|c|c|c|c|c|c|c|c|c|c|c|c|c|c|}
\hline \multirow{2}{*}{ instances } & \multirow{2}{*}{$L B$} & \multicolumn{4}{|c|}{ VND } & \multicolumn{5}{|c|}{ GVNS } & \multicolumn{5}{|c|}{ A-GVNS } \\
\hline & & best.sol & aver.sol & Gap $1_{1}$ & time & best.sol & aver.sol & Gap $1_{1}$ & Gap2 & time & best.sol & aver.sol & Gap $1_{1}$ & Gap2 & time \\
\hline test-30-1 & 6415 & 7116 & 7432.09 & 10.93 & 0.36 & 6415 & 6481.91 & 0.00 & -9.85 & 1.75 & 6415 & 6460.27 & 0.00 & -9.85 & 1.87 \\
\hline test-30-2 & 7186 & 8297 & 8503.91 & 15.46 & 0.16 & 7248 & 7329.91 & 0.86 & -12.64 & 1.7 & 7211 & 7294.36 & 0.35 & -13.09 & 1.84 \\
\hline test-30-3 & 6286 & 7602 & 7726.55 & 20.94 & 0.3 & 6300 & 6376.64 & 0.22 & -17.13 & 1.72 & 6286 & 6349.55 & 0.00 & -17.31 & 2.07 \\
\hline test $-30-4$ & 6348 & 7131 & 7411 & 12.33 & 0.17 & 6366 & 6458.82 & 0.28 & -10.73 & 1.74 & 6348 & 6411.55 & 0.00 & -10.98 & 1.85 \\
\hline test-30-5 & 6239 & 6997 & 7261.91 & 12.15 & 0.13 & 6247 & 6452.45 & 0.13 & -10.72 & 1.81 & 6239 & 6367.27 & 0.00 & -10.83 & 1.8 \\
\hline test-30-6 & 6612 & 7467 & 7787.27 & 12.93 & 0.27 & 6652 & 6805.18 & 0.60 & -10.91 & 1.74 & 6612 & 6742.64 & 0.00 & -11.45 & 1.81 \\
\hline test-30-7 & 7305 & 7918 & 8270.18 & 8.39 & 0.23 & 7312 & 7439.36 & 0.10 & -7.65 & 1.8 & 7305 & 7384.09 & 0.00 & -7.74 & 1.92 \\
\hline test-30-8 & 5907 & 6441 & 6711.09 & 9.04 & 0.23 & 5911 & 6006.27 & 0.07 & -8.23 & 1.74 & 5907 & 5973 & 0.00 & -8.29 & 1.83 \\
\hline test-30-9 & 5988 & 7343 & 7654.55 & 22.63 & 0.29 & 6008 & 6073.55 & 0.33 & -18.18 & 1.82 & 5988 & 6045.18 & 0.00 & -18.45 & 1.87 \\
\hline test-30-10 & 7154 & 8136 & 8401.45 & 13.73 & 0.32 & 7164 & 7625.18 & 0.14 & -11.95 & 1.8 & 7154 & 7479.64 & 0.00 & -12.07 & 1.95 \\
\hline test-30-11 & 7154 & 8136 & 8401.45 & 13.73 & 0.2 & 7164 & 7625.18 & 0.14 & -11.95 & 1.78 & 7154 & 7479.64 & 0.00 & -12.07 & 1.89 \\
\hline test-30-12 & 6173 & 6453 & 6839.36 & 4.54 & 0.29 & 6183 & 6261.09 & 0.16 & -4.18 & 1.75 & 6173 & 6226.91 & 0.00 & -4.34 & 1.83 \\
\hline test-30-13 & 6031 & 6251 & 6571 & 3.65 & 0.22 & 6049 & 6180.82 & 0.30 & -3.23 & 1.74 & 6031 & 6127.55 & 0.00 & -3.52 & 1.95 \\
\hline test-30-14 & 5852 & 7460 & 8127.18 & 27.48 & 0.34 & 5859 & 5939.91 & 0.12 & -21.46 & 1.79 & 5852 & 5909.27 & 0.00 & -21.55 & 1.83 \\
\hline test-30-15 & 6059 & 6257 & 6483.55 & 3.27 & 0.33 & 6066 & 6241.91 & 0.12 & -3.05 & 1.72 & 6059 & 6176.27 & 0.00 & -3.16 & 1.88 \\
\hline test-30-16 & 6669 & 7446 & 7695.18 & 11.65 & 0.17 & 6728 & 6831.36 & 0.88 & -9.64 & 1.75 & 6714 & 6799.09 & 0.67 & -9.83 & 1.96 \\
\hline test-30-17 & 6937 & 7163 & 7253.09 & 3.26 & 0.27 & 6937 & 7003.45 & 0.00 & -3.16 & 1.79 & 6931 & 6968.91 & -0.09 & -3.24 & 1.84 \\
\hline test-30-18 & 6862 & 7961 & 8120.82 & 16.02 & 0.26 & 6888 & 6982.64 & 0.38 & -13.48 & 1.81 & 6862 & 6940.36 & 0.00 & -13.80 & 2.07 \\
\hline test-30-19 & 7469 & 7934 & 8336.09 & 6.23 & 0.25 & 7470 & 7595 & 0.01 & -5.85 & 1.73 & 7469 & 7553.36 & 0.00 & -5.86 & 1.9 \\
\hline test $-30-20$ & 6240 & 6517 & 6788.64 & 4.44 & 0.34 & 6253 & 6426.82 & 0.21 & -4.05 & 1.86 & 6240 & 6357.18 & 0.00 & -4.25 & 1.8 \\
\hline Aver & & & & 11.64 & 0.26 & & & 0.25 & -9.90 & 1.77 & & & 0.05 & -10.09 & 1.89 \\
\hline
\end{tabular}

TABLE IV. THE EXPERIMENTAL RESUlts FOR TEST-30-X WITH $\rho=0.5$

\begin{tabular}{|c|c|c|c|c|c|c|c|c|c|c|c|c|c|c|c|}
\hline \multirow{2}{*}{ instances } & \multirow{2}{*}{$L B$} & \multicolumn{4}{|c|}{ VND } & \multicolumn{5}{|c|}{ GVNS } & \multicolumn{5}{|c|}{ A-GVNS } \\
\hline & & best.sol & aver.sol & Gap $1_{1}$ & time & best.sol & aver.sol & Gap $_{1}$ & Gap2 $_{2}$ & time & best.sol & aver.sol & $G_{1}$ & Gap $2_{2}$ & time \\
\hline test-30-1 & 6415 & 7526 & 7680.55 & 17.32 & 0.17 & 6415 & 6454.91 & 0.00 & -14.76 & 1.7 & 6415 & 6435.55 & 0.00 & -14.76 & 2.03 \\
\hline test-30-2 & 7186 & 7865 & 8003 & 9.45 & 0.18 & 7258 & 7454.09 & 1.00 & -7.72 & 1.77 & 7248 & 7392.18 & 0.86 & -7.84 & 2.1 \\
\hline test-30-3 & 6286 & 6797 & 7152.18 & 8.13 & 0.13 & 6312 & 6423.45 & 0.41 & -7.14 & 1.83 & 6286 & 6377.64 & 0.00 & $\begin{array}{l}-7.52 \\
\end{array}$ & 1.93 \\
\hline test-30-4 & 6348 & 6929 & 7180.64 & 9.15 & 0.34 & 6366 & 6573.64 & 0.28 & -8.13 & 1.78 & 6348 & 6503.45 & 0.00 & $\begin{array}{l}-8.39 \\
\end{array}$ & 1.99 \\
\hline test-30-5 & 6239 & 6966 & 7349.36 & 11.65 & 0.27 & 6247 & 6377.82 & 0.13 & -10.32 & 1.74 & 6239 & 6319.91 & 0.00 & -10.44 & 2.07 \\
\hline test-30-6 & 6612 & 7003 & 7570.55 & 5.91 & 0.22 & 6681 & 6804.36 & 1.04 & -4.60 & 1.63 & 6612 & 6741.64 & 0.00 & -5.58 & 1.78 \\
\hline test-30-7 & 7305 & 7695 & 7891.82 & 5.34 & 0.27 & 7324 & 7451.64 & 0.26 & -4.82 & 1.77 & 7305 & 7413.91 & 0.00 & -5.07 & 1.94 \\
\hline test-30-8 & 5907 & 6486 & 6938.73 & 9.80 & 0.24 & 5911 & 6054.09 & 0.07 & -8.87 & 1.62 & 5907 & 5993.82 & 0.00 & -8.93 & 1.92 \\
\hline test-30-9 & 5988 & 6474 & 6635.73 & 8.12 & 0.27 & 6041 & 6192.91 & 0.89 & -6.69 & 1.65 & 5988 & 6131.55 & 0.00 & -7.51 & 2.07 \\
\hline test-30-10 & 7154 & 8257 & 8407.09 & 15.42 & 0.24 & 7184 & 7531 & 0.42 & -13.00 & 1.63 & 7154 & 7433.27 & 0.00 & -13.36 & 1.99 \\
\hline test-30-11 & 7154 & 8257 & 8407.09 & 15.42 & 0.29 & 7184 & 7531 & 0.42 & -13.00 & 1.75 & 7154 & 7433.27 & 0.00 & -13.36 & 2.03 \\
\hline test-30-12 & 6173 & 6728 & 7550.73 & 8.99 & 0.24 & 6209 & 6346.73 & 0.58 & -7.71 & 1.64 & 6198 & 6299.82 & 0.40 & -7.88 & 1.83 \\
\hline test-30-13 & 6031 & 7031 & 7228.91 & 16.58 & 0.36 & 6057 & 6237.27 & 0.43 & -13.85 & 1.69 & 6031 & 6172.91 & 0.00 & -14.22 & 1.82 \\
\hline test-30-14 & 5852 & 7889 & 8429.27 & 34.81 & 0.16 & 5922 & 6013.82 & 1.20 & -24.93 & 1.8 & 5852 & 5971.36 & 0.00 & -25.82 & 1.81 \\
\hline test-30-15 & 6059 & 6523 & 6753.91 & 7.66 & 0.13 & 6129 & 6259.27 & 1.16 & -6.04 & 1.82 & 6059 & 6195.36 & 0.00 & $\begin{array}{l}-7.11 \\
\end{array}$ & 1.83 \\
\hline test-30-16 & 6669 & 8255 & 8474.27 & 23.78 & 0.13 & 6674 & 6814.73 & 0.07 & -19.15 & 1.79 & 6669 & 6759.09 & 0.00 & -19.21 & 2.02 \\
\hline test-30-17 & 6937 & 7339 & 7558.45 & 5.80 & 0.12 & 6958 & 7075.91 & 0.30 & -5.19 & 1.71 & 6957 & 7024.55 & 0.29 & -5.21 & 2.06 \\
\hline test-30-18 & 6862 & 7476 & 7656.82 & 8.95 & 0.21 & 6890 & 7008.45 & 0.41 & -7.84 & 1.83 & 6862 & 6963.91 & 0.00 & -8.21 & 1.95 \\
\hline test-30-19 & 7469 & 8065 & 8405.36 & 7.98 & 0.22 & 7470 & 7732.82 & 0.01 & -7.38 & 1.72 & 7469 & 7644.27 & 0.00 & $\begin{array}{l}-7.39 \\
\end{array}$ & 1.82 \\
\hline test-30-20 & 6240 & 6322 & 6505.36 & 1.31 & 0.2 & 6280 & 6466.82 & 0.64 & -0.66 & 1.64 & 6240 & 6407.73 & 0.00 & -1.30 & 1.81 \\
\hline Aver & & & & 11.58 & 0.22 & & & 0.49 & -9.59 & 1.73 & & & 0.08 & -9.96 & 1.94 \\
\hline
\end{tabular}

TABLE V. The Experimental Results for Test-30-X with $\rho=0$

\begin{tabular}{|c|c|c|c|c|c|c|c|c|c|c|c|c|c|c|c|}
\hline \multirow{2}{*}{ instances } & \multirow{2}{*}{$L B$} & \multicolumn{4}{|c|}{ VND } & \multicolumn{5}{|c|}{ GVNS } & \multicolumn{5}{|c|}{ A-GVNS } \\
\hline & & best.sol & aver.sol & Gap $_{1}$ & time & best.sol & aver.sol & Gap $_{1}$ & $\mathrm{Gap}_{2}$ & time & best.sol & aver.sol & Gap $_{1}$ & Gap $_{2}$ & time \\
\hline test-30-1 & 6415 & 6505 & 6830.5 & 1.40 & 0.28 & 6415 & 6415 & 0.00 & -1.38 & 0.17 & 6415 & 6415 & 0.00 & -1.38 & 2.04 \\
\hline test-30-2 & 7186 & 7379 & 7561.9 & 2.69 & 0.24 & 7193 & 7193 & 0.10 & -2.52 & 0.18 & 7186 & 7186 & 0.00 & -2.62 & 2.06 \\
\hline test-30-3 & 6286 & 6286 & 6514.4 & 0.00 & 0.28 & 6286 & 6286 & 0.00 & 0.00 & 0.18 & 6286 & 6286 & 0.00 & 0.00 & 1.85 \\
\hline test-30-4 & 6348 & 6872 & 7262.6 & 8.25 & 0.31 & 6348 & 6348 & 0.00 & -7.63 & 0.19 & 6348 & 6348 & 0.00 & -7.63 & 2 \\
\hline test-30-5 & 6239 & 6489 & 6822.2 & 4.01 & 0.32 & 6239 & 6239 & 0.00 & -3.85 & 0.19 & 6239 & 6239 & 0.00 & -3.85 & 2.09 \\
\hline test-30-6 & 6612 & 7017 & 7277.8 & 6.13 & 0.14 & 6612 & 6612 & 0.00 & -5.77 & 0.19 & 6612 & 6612 & 0.00 & -5.77 & 2.05 \\
\hline test-30-7 & 7305 & 7871 & 8270.8 & 7.75 & 0.13 & 7323 & 7323 & 0.25 & -6.96 & 0.18 & 7305 & 7305 & 0.00 & -7.19 & 1.86 \\
\hline test-30-8 & 5907 & 6213 & 6483.3 & 5.18 & 0.26 & 5907 & 5907 & 0.00 & -4.93 & 0.19 & 5907 & 5907 & 0.00 & -4.93 & 1.78 \\
\hline test-30-9 & 5988 & 6386 & 6894.9 & 6.65 & 0.12 & 5988 & 5988 & 0.00 & -6.23 & 0.17 & 5988 & 5988 & 0.00 & -6.23 & 2 \\
\hline test-30-10 & 7154 & 7689 & 8049.8 & 7.48 & 0.22 & 7154 & 7154 & 0.00 & -6.96 & 0.17 & 7154 & 7154 & 0.00 & -6.96 & 1.94 \\
\hline test-30-11 & 7154 & 7689 & 8049.8 & 7.48 & 0.22 & 7154 & 7154 & 0.00 & -6.96 & 0.17 & 7154 & 7154 & 0.00 & -6.96 & 1.87 \\
\hline test-30-12 & 6173 & 6435 & 6726.6 & 4.24 & 0.3 & 6173 & 6173 & 0.00 & -4.07 & 0.19 & 6173 & 6173 & 0.00 & $\begin{array}{l}-4.07 \\
\end{array}$ & 1.81 \\
\hline test-30-13 & 6031 & 6885 & 7197.2 & 14.16 & 0.21 & 6031 & 6031 & 0.00 & -12.40 & 0.18 & 6031 & 6031 & 0.00 & -12.40 & 2.08 \\
\hline test-30-14 & 5852 & 6029 & 6463.2 & 3.02 & 0.19 & 5858 & 5858 & 0.10 & -2.84 & 0.18 & 5852 & 5852 & 0.00 & -2.94 & 1.99 \\
\hline test-30-15 & 6059 & 6878 & 7092.6 & 13.52 & 0.25 & 6059 & 6059 & 0.00 & -11.91 & 0.18 & 6059 & 6059 & 0.00 & -11.91 & 1.83 \\
\hline test-30-16 & 6669 & 7682 & 7966.2 & 15.19 & 0.27 & 6674 & 6674 & 0.07 & -13.12 & 0.17 & 6669 & 6669 & 0.00 & -13.19 & 1.91 \\
\hline test-30-17 & 6937 & 7275 & 7664.2 & 4.87 & 0.22 & 6937 & 6937 & 0.00 & -4.65 & 0.17 & 6937 & 6937 & 0.00 & -4.65 & 1.86 \\
\hline test-30-18 & 6862 & 7260 & 7892 & 5.80 & 0.18 & 6937 & 6937 & 1.09 & -4.45 & 0.2 & 6862 & 6862 & 0.00 & -5.48 & 1.79 \\
\hline test-30-19 & 7469 & 7790 & 8268.9 & 4.30 & 0.13 & 7469 & 7469 & 0.00 & -4.12 & 0.19 & 7469 & 7469 & 0.00 & -4.12 & 2 \\
\hline test-30-20 & 6240 & 7183 & 7310.3 & 15.11 & 0.23 & 6240 & 6240 & 0.00 & -13.13 & 0.2 & 6240 & 6240 & 0.00 & -13.13 & 2.09 \\
\hline Aver & & & & 6.86 & 0.23 & & & 0.08 & -6.19 & 0.18 & & & 0.00 & -6.27 & 1.95 \\
\hline
\end{tabular}


TABLE VI. The EXPERIMENTAL RESUlts For TEST-40-X With $\rho=1$

\begin{tabular}{|c|c|c|c|c|c|c|c|c|c|c|c|c|}
\hline \multirow{2}{*}{ instances } & \multirow{2}{*}{$L B$} & \multicolumn{3}{|c|}{ VND } & \multicolumn{4}{|c|}{ GVNS } & & \multicolumn{3}{|c|}{ A-GVNS } \\
\hline & & best.sol & aver.sol & time & best.sol & aver.sol & Gap 1 & time & best.sol & aver.sol & Gap $_{1}$ & time \\
\hline test-40-1 & 10651 & - & - & - & - & - & - & - & 11730 & 11730 & 10.13 & 8.44 \\
\hline test- $40-2$ & 9541 & - & - & - & 10758 & 10758 & 12.76 & 7.52 & 10758 & 10758 & 12.76 & 8.66 \\
\hline test-40-3 & 9366 & - & - & - & - & - & - & - & 12818 & 12818 & 36.86 & 8.06 \\
\hline test-40-4 & 10210 & - & - & - & 10858 & 10858 & 6.35 & 7.56 & 10858 & 10858 & 6.35 & 8.29 \\
\hline test-40-5 & 9378 & - & - & - & 9788 & 9788 & 4.37 & 7.92 & 9788 & 9788 & 4.37 & 8.19 \\
\hline test- $40-6$ & 9305 & - & - & - & - & - & - & - & 11336 & 11336 & 21.83 & 8.20 \\
\hline test-40-7 & 9241 & - & - & - & 9674 & 9674 & 4.69 & 8.37 & 9674 & 9674 & 4.69 & 8.84 \\
\hline test-40-8 & 9011 & - & - & - & 10343 & 10343 & 14.78 & 7.91 & 10343 & 10343 & 14.78 & 8.64 \\
\hline test- $40-9$ & 10412 & - & - & - & 10869 & 10869 & 4.39 & 7.96 & 10869 & 10869 & 4.39 & 8.67 \\
\hline test $-40-10$ & 9516 & - & - & - & 9606 & 9606 & 0.95 & 7.77 & 9606 & 9606 & 0.95 & 8.77 \\
\hline test $-40-11$ & 9516 & - & - & - & 9606 & 9606 & 0.95 & 7.50 & 9606 & 9606 & 0.95 & 8.80 \\
\hline test $-40-12$ & 9419 & - & - & - & - & - & - & - & 9456 & 9456 & 0.39 & 8.99 \\
\hline test-40-13 & 9995 & - & - & - & 10785 & 10785 & 7.90 & 7.63 & 10785 & 10785 & 7.90 & 8.16 \\
\hline test $-40-14$ & 9975 & - & $\begin{array}{ll}- \\
-\end{array}$ & - & 10348 & 10348 & 3.74 & 8.22 & 10348 & 10348 & 3.74 & 8.02 \\
\hline test $-40-15$ & 9485 & - & - & - & 9559 & 9559 & 0.78 & 7.91 & 9559 & 9559 & 0.78 & 8.65 \\
\hline test $-40-16$ & 10312 & - & - & - & 10877 & 10877 & 5.48 & 8.42 & 10873 & 10873 & 5.44 & 8.52 \\
\hline test-40-17 & 9844 & - & - & - & - & - & - & - & 12191 & 12191 & 23.84 & 8.60 \\
\hline test $-40-18$ & 9790 & - & - & - & - & - & - & - & 12404 & 12404 & 26.70 & 8.40 \\
\hline test $-40-19$ & 9229 & - & - & - & - & - & - & - & 9824 & 9824 & 6.45 & 8.75 \\
\hline test $40-20$ & 8668 & - & - & - & 9280 & 9280 & 7.06 & 7.94 & 9280 & 9280 & 7.06 & 8.37 \\
\hline Aver & & & & & & & & & & & 10.02 & \\
\hline
\end{tabular}

TABLE VII. The EXPERIMENTAL RESUlts For TEST-40-X With $\rho=0.95$

\begin{tabular}{|c|c|c|c|c|c|c|c|c|c|c|c|c|c|c|c|}
\hline \multirow{2}{*}{ instances } & \multirow[b]{2}{*}{$L B$} & \multicolumn{4}{|c|}{ VND } & \multicolumn{5}{|c|}{ GVNS } & \multicolumn{5}{|c|}{ A-GVNS } \\
\hline & & best.sol & aver.sol & Gap $_{1}$ & time & best.sol & aver.sol & Gap $_{1}$ & Gap $_{2}$ & time & best.sol & aver.sol & $G_{a p}$ & Gap $_{2}$ & time \\
\hline test-40-1 & 10651 & 11322 & 11665.36 & 6.30 & 0.31 & 11474 & 11573.27 & 7.73 & 1.34 & 7.31 & 11177 & 11493.36 & 4.94 & -1.28 & 8.47 \\
\hline test-40-2 & 9541 & 9763 & 10015.45 & 2.33 & 0.34 & 9728 & 9824.18 & 1.96 & -0.36 & 7.59 & 9657 & 9777.73 & 1.22 & -1.09 & 7.74 \\
\hline test-40-3 & 9366 & 9751 & 9824.64 & 4.11 & 0.33 & 10350 & 10584.36 & 10.51 & 6.14 & 7.42 & 10284 & 10496.55 & 9.80 & 5.47 & 7.92 \\
\hline test-40-4 & 10210 & 10990 & 11267.55 & 7.64 & 0.32 & 10984 & 11137.55 & 7.58 & -0.05 & 7.61 & 10940 & 11073.64 & 7.15 & -0.45 & 8.16 \\
\hline test-40-5 & 9378 & 10151 & 10378.91 & 8.24 & 0.31 & 10261 & 10347.91 & 9.42 & 1.08 & 7.97 & 10175 & 10299.09 & 8.50 & 0.24 & 8.61 \\
\hline test-40-6 & 9305 & 9746 & 9900.36 & 4.74 & 0.32 & 9729 & 9858.18 & 4.56 & -0.17 & 7.95 & 9693 & 9788.18 & 4.17 & -0.54 & 7.92 \\
\hline test-40-7 & 9241 & 10067 & 10266.27 & 8.94 & 0.33 & 9599 & 9679.64 & 3.87 & -4.65 & 7.39 & 9533 & 9637.27 & 3.16 & -5.30 & 8.12 \\
\hline test- $40-8$ & 9011 & 9776 & 10182.82 & 8.49 & 0.29 & 9346 & 9534.64 & 3.72 & -4.40 & 8.28 & 9320 & 9469.27 & 3.43 & -4.66 & 8.01 \\
\hline test-40-9 & 10412 & 11280 & 11451.09 & 8.34 & 0.3 & 10916 & 11367.64 & 4.84 & -3.23 & 7.59 & 10825 & 11230 & 3.97 & -4.03 & 8.04 \\
\hline test- $40-10$ & 9516 & 10976 & 11336.82 & 15.34 & 0.3 & 9924 & 10102.82 & 4.29 & -9.58 & 7.8 & 9846 & 9998.36 & 3.47 & -10.30 & 7.89 \\
\hline test-40-11 & 9516 & 10976 & 11336.82 & 15.34 & 0.34 & 9924 & 10102.82 & 4.29 & -9.58 & 7.97 & 9846 & 9998.36 & 3.47 & -10.30 & 8.41 \\
\hline test-40-12 & 9419 & 10526 & 11093.91 & 11.75 & 0.3 & 9678 & 10286.18 & 2.75 & -8.06 & 7.83 & 9599 & 10087.36 & 1.91 & -8.81 & 8.5 \\
\hline test-40-13 & 9995 & 10360 & 10830.27 & 3.65 & 0.32 & 10369 & 10866.82 & 3.74 & 0.09 & 7.59 & 10324 & 10704.18 & 3.29 & -0.35 & 8.32 \\
\hline test-40-14 & 9975 & 11714 & 12020.45 & 17.43 & 0.29 & 10470 & 10640.27 & 4.96 & -10.62 & 8.13 & 10443 & 10579.55 & 4.69 & -10.85 & 7.76 \\
\hline test-40-15 & 9485 & 9877 & 10185.64 & 4.13 & 0.33 & 9986 & 10099.18 & 5.28 & 1.10 & 7.9 & 9817 & 10035.27 & 3.50 & -0.61 & 8.36 \\
\hline test-40-16 & 10312 & 11355 & 11518.18 & 10.11 & 0.3 & 10537 & 10929.18 & 2.18 & -7.20 & 7.53 & 10353 & 10789.18 & 0.40 & -8.82 & 8.19 \\
\hline test-40-17 & 9844 & 10676 & 10991.91 & 8.45 & 0.32 & 10398 & 11102.45 & 5.63 & -2.60 & 8.21 & 10241 & 10886.27 & 4.03 & -4.07 & 8.15 \\
\hline test-40-18 & 9790 & 10274 & 10641.09 & 4.94 & 0.33 & 11113 & 11331.45 & 13.51 & 8.17 & 8.29 & 10824 & 11211.64 & 10.56 & 5.35 & 8.58 \\
\hline test-40-19 & 9229 & 9837 & 9999.45 & 6.59 & 0.32 & 9547 & 9989.73 & 3.45 & -2.95 & 8.01 & 9500 & 9847.09 & 2.94 & -3.43 & 8.32 \\
\hline test-40-20 & 8668 & 9680 & 9911.82 & 11.68 & 0.32 & 9384 & 9615.01 & 8.26 & -3.06 & 7.55 & 9147 & 9510.36 & 5.53 & -5.51 & 8.43 \\
\hline Aver & & & & 8.43 & 0.32 & & & 5.63 & -2.43 & 7.80 & & & 4.51 & -3.47 & 8.20 \\
\hline
\end{tabular}

TABLE VIII. THE EXPERIMENTAL RESUlts FOR TEST-40-X With $\rho=0.75$

\begin{tabular}{|c|c|c|c|c|c|c|c|c|c|c|c|c|c|c|c|}
\hline \multirow{2}{*}{ instances } & \multirow{2}{*}{$L B$} & \multicolumn{4}{|c|}{ VND } & \multicolumn{5}{|c|}{ GVNS } & \multicolumn{5}{|c|}{ A-GVNS } \\
\hline & & best.sol & aver.sol & Gap $_{1}$ & time & best.sol & aver.sol & Gap $_{1}$ & Gap $_{2}$ & time & best.sol & aver.sol & Gap $_{1}$ & Gap $_{2}$ & time \\
\hline test- $40-1$ & 10651 & 11135 & 11321.64 & 4.54 & 0.31 & 10653 & 10788.3 & 0.02 & -4.33 & 7.31 & 10651 & 10751.4 & 0.00 & -4.35 & 8.47 \\
\hline test- $40-2$ & 9541 & 10020 & 10239.09 & 5.02 & 0.34 & 9541 & 9618.82 & 0.00 & -4.78 & 7.59 & 9541 & 9587.27 & 0.00 & -4.78 & 7.74 \\
\hline test- $40-3$ & 9366 & 10602 & 11474.27 & 13.20 & 0.33 & 9366 & 9442.82 & 0.00 & -11.66 & 7.42 & 9366 & 9389.09 & 0.00 & -11.66 & 7.92 \\
\hline test-40-4 & 10210 & 10726 & 11076.73 & 5.05 & 0.32 & 10228 & 10350.7 & 0.18 & -4.64 & 7.61 & 10210 & 10310.5 & 0.00 & -4.81 & 8.16 \\
\hline test- $40-5$ & 9378 & 10734 & 11242.36 & 14.46 & 0.31 & 9410 & 9497.82 & 0.34 & -12.33 & 7.97 & 9378 & 9455.09 & 0.00 & -12.63 & 8.61 \\
\hline test-40-6 & 9305 & 11591 & 12135.45 & 24.57 & 0.32 & 9339 & 9412 & 0.37 & -19.43 & 7.95 & 9339 & 9379.09 & 0.37 & -19.43 & 7.92 \\
\hline test-40-7 & 9241 & 11348 & 12219.09 & 22.80 & 0.33 & 9281 & 9414.09 & 0.43 & -18.21 & 7.39 & 9266 & 9367.82 & 0.27 & -18.35 & 8.12 \\
\hline test- $40-8$ & 9011 & 14288 & 14621 & 58.56 & 0.29 & 9011 & 9163.18 & 0.00 & -36.93 & 8.28 & 9011 & 9100 & 0.00 & -36.93 & 8.01 \\
\hline test-40-9 & 10412 & 11780 & 12115.55 & 13.14 & 0.3 & 10440 & 10602.4 & 0.27 & -11.38 & 7.59 & 10418 & 10546.5 & 0.06 & -11.56 & 8.04 \\
\hline test $-40-10$ & 9516 & 10093 & 10882.82 & 6.06 & 0.3 & 9577 & 9618.64 & 0.64 & -5.11 & 7.8 & 9516 & 9591.36 & 0.00 & -5.72 & 7.89 \\
\hline test-40-11 & 9516 & 10093 & 10882.82 & 6.06 & 0.34 & 9577 & 9618.64 & 0.64 & -5.11 & 7.97 & 9516 & 9591.36 & 0.00 & -5.72 & 8.41 \\
\hline test- $40-12$ & 9419 & 11733 & 12735.91 & 24.57 & 0.3 & 9443 & 9565.55 & 0.25 & -19.52 & 7.83 & 9440 & 9517.91 & 0.22 & -19.54 & 8.5 \\
\hline test-40-13 & 9995 & 11522 & 11949.18 & 15.28 & 0.32 & 10025 & 10241.7 & 0.30 & -12.99 & 7.59 & 9995 & 10162.6 & 0.00 & -13.25 & 8.32 \\
\hline test-40-14 & 9975 & 15264 & 15755 & 53.02 & 0.29 & 9984 & 10114 & 0.09 & -34.59 & 8.13 & 9975 & 32.6 & 0.00 & -34.65 & 7.76 \\
\hline test-40-15 & 9485 & 10178 & 10555.64 & 7.31 & 0.33 & 9485 & 9569.18 & 0.00 & -6.81 & 7.9 & 9485 & 9542.55 & 0.00 & -6.81 & 8.36 \\
\hline test-40-16 & 10312 & 11777 & 12135.55 & 14.21 & 0.3 & 10465 & 10569.4 & 1.48 & -11.14 & 7.53 & 10441 & 10501.7 & 1.25 & -11.34 & 8.19 \\
\hline test $-40-17$ & 9844 & 10705 & 11068.27 & 8.75 & 0.32 & 9852 & 9954.27 & 0.08 & -7.97 & 8.21 & 9844 & 9911.55 & 0.00 & -8.04 & 8.15 \\
\hline test-40-18 & 9790 & 13440 & 13697 & 37.28 & 0.33 & 9806 & 9847 & 0.16 & -27.04 & 8.29 & 9790 & 9826.55 & 0.00 & -27.16 & 8.58 \\
\hline test-40-19 & 9229 & 13897 & 14302.45 & 50.58 & 0.32 & 9362 & 9442.09 & 1.44 & -32.63 & 8.01 & 9267 & 9402.36 & 0.41 & -33.32 & 8.32 \\
\hline test $-40-20$ & 8668 & 10040 & 10607.45 & 15.83 & 0.32 & 8668 & 8736.45 & 0.00 & -13.67 & 7.55 & 8668 & 8704.55 & 0.00 & -13.67 & 8.43 \\
\hline Aver & & & & 20.01 & 0.32 & & & 0.34 & -15.01 & 7.80 & & & 0.13 & -15.19 & 8.20 \\
\hline
\end{tabular}


TABLE IX. The EXPERIMENTAL RESUlts FOR TEST-40-X With $\rho=0.5$

\begin{tabular}{|c|c|c|c|c|c|c|c|c|c|c|c|c|c|c|c|}
\hline \multirow{2}{*}{ instances } & \multirow{2}{*}{$L B$} & \multicolumn{4}{|c|}{ VND } & \multicolumn{5}{|c|}{ GVNS } & \multicolumn{5}{|c|}{ A-GVNS } \\
\hline & & best.sol & aver.sol & Gap $_{1}$ & time & best.sol & aver.sol & Gap 1 & Gap $2_{2}$ & time & best.sol & aver.sol & Gap $1_{1}$ & Gap $2_{2}$ & time \\
\hline test-40-1 & 0651 & 11143 & 11489.45 & 4.62 & 0.32 & 10683 & 10810 & 0.30 & -4.13 & 7.93 & 10662 & 10756.2 & 0.10 & -4.32 & 8.06 \\
\hline test-40-2 & 541 & 950 & 10074.64 & 4.29 & 0.3 & 9541 & 9633.27 & 0.00 & -4.11 & 7.87 & 9541 & 9601.55 & 0.00 & -4.11 & 7.6 \\
\hline test-40-3 & 6 & 0143 & 10256.91 & 8.30 & 0.31 & 9454 & 9601.64 & 0.94 & -6.79 & 7.95 & 9397 & 9534.82 & .33 & -7.35 & 7.62 \\
\hline test- $40-4$ & 210 & 624 & 10907.09 & 4.05 & 0.32 & 10258 & 10324.3 & 0.47 & -3.45 & 7.11 & 10210 & 10287.4 & 00 & 3.90 & 8.16 \\
\hline test-40-5 & 78 & 0503 & 10671.09 & 12.00 & 0.31 & 9430 & 9560.27 & 0.55 & -10.22 & 7.38 & 9410 & 9511.36 & 0.34 & -10.41 & 7.88 \\
\hline test-40-6 & 9305 & 9624 & 9827.36 & 3.43 & 0.31 & 9325 & 9471.73 & 0.21 & -3.11 & 7.42 & 9305 & 9413 & 0.00 & -3.31 & 7.51 \\
\hline test- &  & 198 & 11710.36 & 21.18 & 0.29 & 9288 & 9376 & 0.51 & -17.06 & 7.5 & 9281 & 9342.55 & .43 & -17.12 & 8.03 \\
\hline test-4 & & 072 & 10303.18 & 11.77 & 0.29 & 9011 & 9971.73 & 0.00 & -10.53 & 7.29 & 90 & 8926.45 & 0 & -10.53 & 7.48 \\
\hline test-40-9 & 10412 & 036 & 15523.91 & 44.41 & 0.32 & 10431 & 10532.6 & 0.18 & -30.63 & 7.33 & 10412 & 10500.1 & 0.00 & -30.75 & 7.56 \\
\hline & & 149 & 11603.64 & 17.16 & 0.31 & 9516 & 9598.73 & 0.00 & -14.65 & 7.92 & 9516 & 9568 & 0.00 & -14.65 & 8.06 \\
\hline test-40-11 & 16 & 149 & 11603.64 & 17.16 & 0.32 & 951 & 9598.73 & 0.00 & -14.65 & 8.09 & 951 & 9568 & .00 & -14.65 & 7.89 \\
\hline test $-40-12$ & 9419 & 0127 & 10548.36 & 7.52 & 0.31 & 9440 & 9526.27 & 0.22 & -6.78 & 7.15 & 9419 & 9485.45 & 0.00 & -6.99 & 8.09 \\
\hline & & 33 & 140 & 34.40 & 0.31 & 10000 & 102 & 0.05 & -25.56 & 8.09 & 9995 & & & -25.59 & 7.93 \\
\hline test- & 75 & 77 & 103 & 1.72 & 0.3 & 9984 & 1000 & 0.09 & -1.61 & 7.57 & 9975 & 9.4 & 0 & -1.70 & 7.61 \\
\hline test $-40-15$ & 9485 & 10692 & 11404.45 & 12.73 & 0.31 & 9575 & 9634.82 & 0.95 & -10.45 & 7.9 & 9539 & 9607.82 & 0.57 & -10.78 & 7.84 \\
\hline test- $40-16$ & 312 & 14210 & 14443.73 & 37.80 & 0.3 & 10344 & 10542.3 & 0.31 & -27.21 & 7.59 & 10312 & & 0.00 & -27.43 & 7.41 \\
\hline & & & & 10.38 & & & & 0.64 & -8.83 & 8.06 & 9844 & 10000.5 & 0 & -9.41 & 7.64 \\
\hline test- $40-18$ & 9790 & 11050 & 11544.55 & 12.87 & 0.31 & 9957 & 10039.6 & 1.71 & -9.89 & 7.25 & 9845 & 9992 & 0.56 & -10.90 & 7.91 \\
\hline test $-40-19$ & 229 & 10569 & 10865.82 & 14.52 & 0.29 & 9291 & 9346.36 & 0.67 & -12.09 & 7.79 & 9229 & 9316.45 & 0.00 & -12.68 & 8.14 \\
\hline test-40 & 08 & 9981 & 10138.64 & 15.15 & 0.31 & 8668 & 8785.55 & 0.00 & -13.15 & 8.04 & 866 & .82 & .00 & -13.15 & 7.53 \\
\hline Aver & & & & 14.77 & 0.31 & & & 0.39 & -11.74 & 7.66 & & & 0.12 & -11.99 & 7.80 \\
\hline
\end{tabular}

TABLE X. The EXPERIMENTAL RESUlts FOR TEST-40-X With $\rho=0$

\begin{tabular}{|c|c|c|c|c|c|c|c|c|c|c|c|c|c|c|c|}
\hline \multirow{2}{*}{ instances } & \multirow{2}{*}{$L B$} & \multicolumn{4}{|c|}{ VND } & \multicolumn{5}{|c|}{ GVNS } & \multicolumn{5}{|c|}{ A-GVNS } \\
\hline & & best.sol & aver.sol & Gap $_{1}$ & time & best.sol & aver.sol & Gap $_{1}$ & Gap $_{2}$ & time & best.sol & aver.sol & Gap $_{1}$ & $\mathrm{Gap}_{2}$ & time \\
\hline test-40-1 & 10651 & 11085 & 11196.1 & 4.07 & 0.3 & 10651 & 10702.3 & 0.00 & -3.92 & 7.93 & 10651 & 10651 & 0.00 & -3.92 & 7.87 \\
\hline test-40-2 & 9541 & 0367 & 10519.9 & 8.66 & 0.31 & 9541 & 9593.8 & 0.00 & -7.97 & 7.08 & 9541 & 9541 & 0.00 & -7.97 & 8.02 \\
\hline test-40-3 & 9366 & 9925 & 10118.7 & 5.97 & 0.29 & 9366 & 9405.1 & 0.00 & -5.63 & 7.79 & 9366 & 9366 & 0.00 & -5.63 & 7.68 \\
\hline test-40-4 & 21 & 1851 & 13162.3 & 16.07 & 0.31 & 10210 & 10278.6 & 0.00 & -13.85 & 7.19 & 10210 & 10210 & .00 & -13.85 & 7.71 \\
\hline test-40-5 & 9378 & 9612 & 10137.6 & 2.50 & 0.3 & 9378 & 9467.5 & 0.00 & -2.43 & 7.33 & 9378 & 9378 & 0.00 & -2.43 & 7.21 \\
\hline test-40-6 & 9305 & 9609 & 9774 & 3.27 & 0.31 & 9305 & 9338.9 & 0.00 & -3.16 & 7.32 & 9305 & 9305 & 0.00 & -3.16 & 7.34 \\
\hline test- $40-7$ & 4 & 11010 & 13378.8 & 19.14 & 0.31 & 9241 & 9282.6 & 0.00 & -16.07 & 6.87 & 9241 & 9241 & 0.00 & -16.07 & 7.83 \\
\hline test- $40-8$ & 901 & 9454 & 10026.1 & 4.92 & 0.3 & 9011 & 9039.5 & 0.00 & -4.69 & 7.77 & 9011 & 9011 & 0.00 & -4.69 & 7.14 \\
\hline test-40-9 & 10412 & 11239 & 11509.1 & 7.94 & 0.3 & 10412 & 10457.2 & 0.00 & -7.36 & 7.33 & 10412 & 10412 & 0.00 & -7.36 & 7.41 \\
\hline test $-40-10$ & 051 & 434 & 15975.6 & 30.66 & 0.31 & 9516 & 9573.6 & 0.00 & -23.47 & 6.99 & 9516 & 9516 & 0.00 & -23.47 & 7.55 \\
\hline test $-40-11$ & 951 & 12434 & 15975.6 & 30.66 & 0.31 & 9516 & 9573.6 & 0.00 & -23.47 & 6.85 & 9516 & 9516 & 0.00 & -23.47 & 7.38 \\
\hline test $-40-12$ & 9419 & 10572 & 11247.5 & 12.24 & 0.3 & 9419 & 9445.8 & 0.00 & -10.91 & 7.5 & 9419 & 9419 & 0.00 & -10.91 & 7.16 \\
\hline test-40- & 3 & 163 & 12082.4 & 11.69 & 0.3 & 9995 & 10036.5 & 0.00 & -10.46 & 7.03 & 9995 & 9995 & 0.00 & -10.46 & 7.55 \\
\hline test-40-1 & 9975 & 321 & 10931.6 & 3.47 & 0.31 & 9975 & 9996.6 & 0.00 & -3.35 & 7.06 & 9975 & 9975 & 0.00 & -3.35 & 7.4 \\
\hline test- $40-15$ & 9485 & 9748 & 10076.3 & 2.77 & 0.29 & 9485 & 9540.7 & 0.00 & -2.70 & 7.17 & 9485 & 9485 & 0.00 & -2.70 & 8.00 \\
\hline test-40-16 & 10312 & 11058 & 11907 & 7.23 & 0.31 & 10312 & 10413.5 & 0.0 & -6.75 & 7.18 & 10312 & 10312 & 0.00 & -6.75 & 7.66 \\
\hline & 9844 & & 13205.3 & 23.84 & 0.29 & 9844 & & 0. & -19.25 & 7.63 & 9844 & 9844 & - & -19.25 & 7.52 \\
\hline test $-40-18$ & 9790 & 10913 & 11290.7 & 11.47 & 0.31 & 9790 & 9838.8 & 0.00 & -10.29 & 7.24 & 9790 & 9790 & 0.00 & -10.29 & 7.12 \\
\hline test $-40-19$ & 9229 & 10327 & 10734.1 & 11.90 & 0.29 & 9229 & 9322.3 & 0.00 & -10.63 & 6.83 & 9229 & 9229 & 0.00 & -10.63 & 7.44 \\
\hline test $-40-20$ & 8668 & 8731 & 9343.4 & 0.73 & 0.31 & 8668 & 8722.8 & 0.00 & -0.72 & 6.91 & 8668 & 8668 & 0.00 & -0.72 & 7.81 \\
\hline Aver & & & & 10.96 & 0.30 & & & 0.00 & -9.35 & 7.37 & & & 0.00 & -9.35 & 7.54 \\
\hline
\end{tabular}

$L B$ : Since $\rho=0$, the Q-TRP becomes to the TRP. The optimal solution of the TRP found by using the exact algorithm in [2] are the lower bound of the optimal solution of the Q-TRP.

TABLE XI. The EXPERIMENTAL RESUlts FOR TEST-50-X With $\rho=1$

\begin{tabular}{|l|c|c|c|c|c|c|c|c|c|}
\hline \multirow{2}{*}{ instances } & \multicolumn{3}{|c|}{ VND } & \multicolumn{3}{|c|}{ GVNS } & \multicolumn{3}{c|}{ A-GVNS } \\
\cline { 2 - 10 } & best.sol & aver.sol & time & best.sol & aver.sol & time & best.sol & aver.sol & Time \\
\hline test-50-1 & - & - & - & - & - & - & 14158 & 14158 & 28.6 \\
\hline test-50-2 & - & - & - & 14633 & 14633 & 26.3 & 14633 & 14633 & 28.7 \\
\hline test-50-3 & - & - & - & - & - & - & 14632 & 14632 & 27.8 \\
\hline test-50-4 & - & - & - & - & - & - & 16600 & 16600 & 27.6 \\
\hline test-50-5 & - & - & - & - & - & - & 16113 & 16113 & 27.0 \\
\hline test-50-6 & - & - & - & - & - & - & 15897 & 15897 & 27.2 \\
\hline test-50-7 & - & - & - & 15572 & 15572 & 24.9 & 14907 & 14907 & 27.3 \\
\hline test-50-8 & - & - & - & - & - & - & 16796 & 16796 & 26.0 \\
\hline test-50-9 & - & - & - & 15354 & 15354 & 25.4 & 15354 & 15354 & 27.2 \\
\hline test-50-10 & - & - & - & - & - & - & 16861 & 16861 & 28.0 \\
\hline test-50-11 & - & - & - & - & - & - & 14583 & 14583 & 28.1 \\
\hline test-50-12 & - & - & - & - & - & - & 15612 & 15612 & 26.4 \\
\hline test-50-13 & - & - & - & - & - & - & 14249 & 14249 & 26.3 \\
\hline test-50-14 & - & - & - & - & - & - & 15731 & 15731 & 26.0 \\
\hline test-50-15 & - & - & - & - & - & - & 16884 & 16884 & 27.1 \\
\hline test-50-16 & - & - & - & 15827 & 15827 & 24.9 & 13874 & 13874 & 27.7 \\
\hline test-50-17 & - & - & - & 14457 & 14457 & 26.4 & 13155 & 13155 & 28.1 \\
\hline test-50-18 & - & - & - & 14459 & 14459 & 24.9 & 13182 & 13182 & 26.0 \\
\hline test-50-19 & - & - & - & 15662 & 15662 & 24.4 & 15662 & 15662 & 28.9 \\
\hline test-50-20 & - & - & - & - & - & - & 14407 & 14407 & 27.6 \\
\hline
\end{tabular}


TABLE XII. THE EXPERIMENTAL RESUlts FOR TEST-50-X With $\rho=0.95$

\begin{tabular}{|c|c|c|c|c|c|c|c|c|c|c|c|}
\hline \multirow{2}{*}{ instances } & \multicolumn{3}{|c|}{ VND } & \multicolumn{4}{|c|}{ GVNS } & \multicolumn{4}{|c|}{ A-GVNS } \\
\hline & best.sol & aver.sol & time & best.sol & aver.sol & Gap $_{2}$ & time & best.sol & aver.sol & $\mathrm{Gap}_{2}$ & time \\
\hline test-50-1 & 15097 & 15660.45 & 1.92 & 13524 & 13790 & -10.42 & 23.9 & 13256 & 13673.27 & -12.19 & 24.7 \\
\hline test-50-2 & 17535 & 17936.36 & 2.13 & 12426 & 12491.73 & -29.14 & 23.3 & 12305 & 12452.18 & -29.83 & 24.1 \\
\hline test-50-3 & 14109 & 14708.82 & 2.32 & 13497 & 13636.55 & -4.34 & 23.3 & 13352 & 13568 & -5.37 & 25.2 \\
\hline test-50-4 & 17491 & 17921.64 & 2.51 & 14422 & 14727 & -17.55 & 24.6 & 14325 & 14590.09 & -18.10 & 23.3 \\
\hline test-50-5 & 14419 & 14531.09 & 2.4 & 14348 & 14871.36 & -0.49 & 24 & 14194 & 14663.45 & -1.56 & 24.2 \\
\hline test-50-6 & 15166 & 15590.09 & 2.41 & 15058 & 15433.09 & -0.71 & 23.1 & 14784 & 15303.82 & -2.52 & 24.4 \\
\hline test-50-7 & 15192 & 15469.55 & 2.02 & 15418 & 15825.55 & 1.49 & 23.3 & 15249 & 15686.18 & 0.38 & 23.8 \\
\hline test-50-8 & 14988 & 15801.27 & 2.55 & 14898 & 15102.91 & -0.60 & 22.1 & 14783 & 15015.09 & -1.37 & 24.2 \\
\hline test-50-9 & 17273 & 18060.91 & 2.5 & 14977 & 15218.45 & -13.29 & 23.7 & 14956 & 15093.73 & -13.41 & 24.1 \\
\hline test-50-10 & 14518 & 15021.91 & 2.03 & 13718 & 13989.09 & -5.51 & 23.8 & 13625 & 13885.91 & -6.15 & 24.5 \\
\hline test-50-11 & 14518 & 15021.91 & 2.46 & 13718 & 13989.09 & -5.51 & 24 & 13625 & 13885.91 & -6.15 & 25 \\
\hline test-50-12 & 15687 & 16114.45 & 2.22 & 14476 & 14932.73 & -7.72 & 23.9 & 14441 & 14796.73 & -7.94 & 25.3 \\
\hline test-50-13 & 13079 & 13326.91 & 2.52 & 12496 & 12716.73 & -4.46 & 22.7 & 12452 & 12636.09 & -4.79 & 24.7 \\
\hline test-50-14 & 14205 & 1512 & 2.17 & 13621 & 6.91 & -4.11 & 22.3 & 13515 & 13653.27 & -4.86 & 24.9 \\
\hline test-50-15 & 13610 & 14132.73 & 2.09 & 14070 & 14323.55 & 3.38 & 22.2 & 13915 & 14204 & 2.24 & 25.7 \\
\hline test-50-16 & 16585 & 16970.73 & 2.57 & 14127 & 15262.27 & -14.82 & 23.3 & 13940 & 14896.18 & -15.95 & 23 \\
\hline test-50-17 & 13643 & 13933.91 & 2.6 & 13138 & 13361.82 & -3.70 & 24.2 & 13095 & 13284.64 & -4.02 & 23.7 \\
\hline test-50-18 & 14329 & 14582.55 & 2.26 & 13748 & 14026.09 & -4.05 & 23.9 & 13553 & 13915.73 & -5.42 & 25.8 \\
\hline test-50-19 & 14959 & 15452.36 & 2.06 & 14781 & 14992.91 & -1.19 & 23.5 & 14721 & 14910.45 & -1.59 & 25.4 \\
\hline test-50-20 & 15046 & 15258.73 & 2.34 & 14527 & 14623.82 & -3.45 & 22.3 & 14370 & 14569.09 & -4.49 & 24.7 \\
\hline Aver & & & 2.30 & & & -6.31 & 7.37 & & & -7.15 & 24.54 \\
\hline
\end{tabular}

TABLE XIII. THE EXPERIMENTAL RESUlTS FOR TEST-50-X WITH $\rho=0.75$

\begin{tabular}{|c|c|c|c|c|c|c|c|c|c|c|c|}
\hline \multirow{2}{*}{ instances } & \multicolumn{3}{|c|}{ VND } & \multicolumn{4}{|c|}{ GVNS } & \multicolumn{4}{|c|}{ A-GVNS } \\
\hline & best.sol & aver.sol & time & best.sol & aver.sol & Gap2 $_{2}$ & time & best.sol & aver.sol & Gap $2_{2}$ & time \\
\hline test-50-1 & 13841 & 14083.18 & 2.47 & 12801 & 13013.45 & -7.51 & 23.1 & 12695 & 12921.73 & -8.28 & 27.9 \\
\hline test-50-2 & 12783 & 13119.09 & 2.27 & 11953 & 12071.82 & -6.49 & 25.7 & 11925 & 12029.73 & -6.71 & 27.9 \\
\hline test-50-3 & 14700 & 15256.09 & 2.6 & 12682 & 12763.18 & -13.73 & 23.8 & 12630 & 12716.36 & -14.08 & 26.3 \\
\hline test-50-4 & 15920 & 16322.91 & 2.26 & 14004 & 14089.36 & -12.04 & 24.1 & 13979 & 14055.82 & -12.19 & 27 \\
\hline test-50-5 & 19703 & 19915.27 & 2.12 & 13909 & 14042.64 & -29.41 & 24.7 & 13881 & 13990 & -29.55 & 26.5 \\
\hline test-50-6 & 14804 & 15217 & 2.07 & 13974 & 14056.36 & -5.61 & 24.4 & 13885 & 14009.64 & -6.21 & 25.9 \\
\hline test-50-7 & 19768 & 21799 & 2.39 & 13739 & 13900.45 & -30.50 & 25.5 & 13712 & 13834.55 & -30.64 & 25.9 \\
\hline test-50-8 & 14973 & 15601.73 & 2.1 & 14291 & 14354.09 & -4.55 & 25.1 & 14290 & 14329.36 & -4.56 & 25.9 \\
\hline test-50-9 & 15242 & 15390.27 & 2.63 & 14754 & 14864.64 & -3.20 & 25.1 & 14716 & 14822.55 & -3.45 & 25 \\
\hline test-50-10 & 13829 & 141 & 2.33 & 13381 & 134 & -3.24 & 24.1 & 13354 & 134 & -3.43 & 26.6 \\
\hline test-50-11 & 13829 & 14166.18 & 2.21 & 13381 & 13472.18 & -3.24 & 24.6 & 13354 & 13436.82 & -3.43 & 25.9 \\
\hline test-50-12 & 18512 & 18871.36 & 2.25 & 13755 & 13861.82 & -25.70 & 24.9 & 13730 & 13815.27 & -25.83 & 26.5 \\
\hline test-50-13 & 18263 & 18502.82 & 2.52 & 12308 & 12469.09 & -32.61 & 24.7 & 12306 & 12420.82 & -32.62 & 25.9 \\
\hline test-50-14 & 16590 & 16820.18 & 2.68 & 13131 & 13270.45 & -20.85 & 23.5 & 13078 & 13228.82 & -21.17 & 25.6 \\
\hline test-50-15 & 19048 & 19585.82 & 2.23 & 13127 & 13227.55 & -31.08 & 25.9 & 13101 & 13187.18 & -31.22 & 27.7 \\
\hline test-50-16 & 22257 & 22602.36 & 2.02 & 13667 & 1370 & -38.59 & 25.7 & 13651 & 1368 & -38.67 & 27.5 \\
\hline test-50-17 & 13087 & 13283.82 & 2.69 & 12847 & 12985.27 & -1.83 & 24.2 & 12842 & 12925.45 & -1.87 & 25 \\
\hline test-50-18 & 21169 & 21831 & 2.48 & 12698 & 12823.91 & -40.02 & 25.3 & 12684 & 12765.27 & -40.08 & 27.7 \\
\hline test-50-19 & 16734 & 17059 & 2.34 & 14443 & 14646.18 & -13.69 & 25.4 & 14404 & 14558.18 & -13.92 & 27.1 \\
\hline test-50-20 & 15016 & 15460.55 & 2.42 & 13623 & 13740.91 & -9.28 & 24.2 & 13606 & 13695 & -9.39 & 26.7 \\
\hline Aver & & & 2.35 & & & -16.66 & 24.7 & & & -16.87 & 26.53 \\
\hline
\end{tabular}


TABLE XIV. The EXPERIMENTAL ResUlts For Test-50-X With $\rho=0.5$

\begin{tabular}{|c|c|c|c|c|c|c|c|c|c|c|c|}
\hline \multirow{2}{*}{ instances } & \multicolumn{3}{|c|}{ VND } & \multicolumn{4}{|c|}{ GVNS } & \multicolumn{4}{|c|}{ A-GVNS } \\
\hline & best.sol & aver.sol & time & best.sol & aver.sol & Gap $2_{2}$ & time & best.sol & aver.sol & Gap $2_{2}$ & time \\
\hline test-50-1 & 14306 & 14751.09 & 2.47 & 12869 & 13002.73 & -10.04 & 23.1 & 12746 & 12924.45 & -10.90 & 27.9 \\
\hline test-50-2 & 12581 & 13114.73 & 2.27 & 11939 & 12143.55 & -5.10 & 25.7 & 11925 & 12060.91 & -5.21 & 27.9 \\
\hline test-50-3 & 18448 & 18812.55 & 2.6 & 12753 & 12958.73 & -30.87 & 23.8 & 12610 & 12887.45 & -31.65 & 26.3 \\
\hline test-50-4 & 22125 & 22448.64 & 2.26 & 13993 & 14056.36 & -36.75 & 24.1 & 13949 & 14025.45 & -36.95 & 27 \\
\hline test-50-5 & 15066 & 15412.64 & 2.12 & 14052 & 14189.27 & -6.73 & 24.7 & 14037 & 14141.64 & -6.83 & 26.5 \\
\hline test-50-6 & 15900 & 16725 & 2.07 & 13918 & 14009 & -12.47 & 24.4 & 13905 & 13972.09 & -12.55 & 25.9 \\
\hline test-50-7 & 26742 & 27488.27 & 2.39 & 13739 & 13821 & -48.62 & 25.5 & 13739 & 13795.82 & -48.62 & 25.9 \\
\hline test-50-8 & 14801 & 15540.91 & 2.1 & 14292 & 14433.27 & -3.44 & 25.1 & 14290 & 14379.18 & -3.45 & 25.9 \\
\hline test-50-9 & 23731 & 24268.18 & 2.63 & 14587 & 14729.27 & -38.53 & 25.1 & 14479 & 14669.36 & -38.99 & 25 \\
\hline test-50-10 & 13982 & 14402.82 & 2.33 & 13381 & 13493 & -4.30 & 24.1 & 13354 & 13447.91 & -4.49 & 26.6 \\
\hline test-50-11 & 13982 & 14402.82 & 2.21 & 13381 & 13493 & -4.30 & 24.6 & 13354 & 13447.91 & -4.49 & 25.9 \\
\hline test-50-12 & 15235 & 15639.82 & 2.25 & 13793 & 13992.91 & -9.47 & 24.9 & 13792 & 13932.73 & -9.47 & 26.5 \\
\hline test-50-13 & 18515 & 19144.82 & 2.52 & 12308 & 12433.18 & -33.52 & 24.7 & 12306 & 12389.73 & -33.53 & 25.9 \\
\hline test-50-14 & 16811 & 1698 & 2.68 & 13068 & 13164.55 & -22.27 & 23.5 & 13065 & 4.18 & -22.28 & 25.6 \\
\hline test-50-15 & 14097 & 14781.82 & 2.23 & 13209 & 13274.73 & -6.30 & 25.9 & 13155 & 13245.45 & -6.68 & 27.7 \\
\hline test-50-16 & 19852 & 20921.55 & 2.02 & 13667 & 13748.09 & -31.16 & 25.7 & 13651 & 13719.91 & -31.24 & 27.5 \\
\hline test-50-17 & 15464 & 16994.64 & 2.69 & 12881 & 13003.09 & -16.70 & 24.2 & 12872 & 12929.45 & -16.76 & 25 \\
\hline test-50-18 & 14661 & 14864.55 & 2.48 & 12684 & 12795.82 & -13.48 & 25.3 & 12680 & 12757.45 & -13.51 & 27.7 \\
\hline test-50-19 & 18940 & 19361.91 & 2.34 & 14145 & 14259.27 & -25.32 & 25.4 & 14124 & 14205.91 & -25.43 & 27.1 \\
\hline test-50-20 & 15138 & 15735.55 & 2.42 & 13743 & 13875.45 & -9.22 & 24.2 & 13606 & 13807.64 & -10.12 & 26.7 \\
\hline Aver & & & 2.35 & & & -18.43 & 24.7 & & & -18.66 & 26.53 \\
\hline
\end{tabular}

TABLE XV. The EXPERIMENTAL RESUlts For TEST-50-X With $\rho=0$

\begin{tabular}{|c|c|c|c|c|c|c|c|c|c|c|c|}
\hline \multirow{2}{*}{ instances } & \multicolumn{3}{|c|}{ VND } & \multicolumn{4}{|c|}{ GVNS } & \multicolumn{4}{|c|}{ A-GVNS } \\
\hline & best.sol & aver.sol & time & best.sol & aver.sol & Gap2 & time & best.sol & aver.sol & $\operatorname{Gap}_{2}$ & time \\
\hline test-50-1 & 14049 & 15118.1 & 1.82 & 12695 & 12879.27 & -9.64 & 22.3 & 12695 & 12801.2 & -9.64 & 24.7 \\
\hline test-50-2 & 15881 & 18102.7 & 2.36 & 11925 & 12007.09 & -24.91 & 21.4 & 11925 & 11966.2 & -24.91 & 24.1 \\
\hline test-50-3 & 14976 & 17840.7 & 1.95 & 12740 & 12808.27 & -14.93 & 21.6 & 12686 & 12762.5 & -15.29 & 25.2 \\
\hline test-50-4 & 17086 & 18990.3 & 2.15 & 13973 & 14088.45 & -18.22 & 21.6 & 13961 & 14028.1 & -18.29 & 23.3 \\
\hline test-50-5 & 14097 & 14510.9 & 1.81 & 13957 & 14099.18 & -0.99 & 23.5 & 13924 & 14014.4 & -1.23 & 24.2 \\
\hline test-50-6 & 14683 & 14915.7 & 1.98 & 13858 & 14014.27 & -5.62 & 21.2 & 13841 & 13959.2 & -5.73 & 24.4 \\
\hline test-50-7 & 17594 & 21650.4 & 2.11 & 13798 & 13914.18 & -21.58 & 23.5 & 13739 & 13840.5 & -21.91 & 23.8 \\
\hline test-50-8 & 14850 & 15151.5 & 2.5 & 14290 & 14356.55 & -3.77 & 23.2 & 14290 & 14316.7 & -3.77 & 24.2 \\
\hline test-50-9 & 17706 & 21430 & 2.39 & 14822 & 14919.18 & -16.29 & 23.3 & 14762 & 14853.3 & -16.63 & 24.1 \\
\hline test-50-10 & 13844 & 14141.3 & 1.97 & 13381 & 13544.91 & -3.34 & 22.5 & 13354 & 13443.1 & -3.54 & 24.5 \\
\hline test-50-11 & 13844 & 14141.3 & 1.93 & 13381 & 13544.91 & -3.34 & 21.2 & 13354 & 13443.1 & -3.54 & 25 \\
\hline test-50-12 & 16831 & 18960.4 & 2.23 & 13729 & 13821.18 & -18.43 & 22 & 13721 & 13776.5 & -18.48 & 25.3 \\
\hline test-50-13 & 13392 & 13789 & 2.47 & 12306 & 12411.73 & -8.11 & 23.4 & 12306 & 12364.1 & -8.11 & 24.7 \\
\hline test-50-14 & 14580 & 15316.8 & 2.35 & 13065 & 13104 & -10.39 & 21.4 & 13065 & 13080.3 & -10.39 & 24.9 \\
\hline test-50-15 & 13919 & 14105.4 & 2.33 & 13371 & 13453.73 & -3.94 & 21.8 & 13332 & 13402.7 & -4.22 & 25.7 \\
\hline test-50-16 & 15056 & 16270.8 & 2.41 & 13651 & 13734.55 & -9.33 & 23.3 & 13651 & 13700.8 & -9.33 & 23 \\
\hline test-50-17 & 13658 & 14176.7 & 2.4 & 12990 & 13051.27 & -4.89 & 21.3 & 12842 & 12989.4 & -5.97 & 23.7 \\
\hline test-50-18 & 12967 & 13232.2 & 2.25 & 12688 & 12778.09 & -2.15 & 21.3 & 12688 & 12728.2 & -2.15 & 25.8 \\
\hline test-50-19 & 15102 & 15399.8 & 2.07 & 14291 & 14413 & -5.37 & 21.3 & 14260 & 14356.4 & -5.58 & 25.4 \\
\hline test-50-20 & 14299 & 15254.2 & 2.26 & 13766 & 13869.91 & -3.73 & 21.9 & 13743 & 13822.7 & -3.89 & 24.7 \\
\hline Aver & & & 2.19 & & & -9.45 & 22.15 & & & -9.63 & 24.54 \\
\hline
\end{tabular}


TABLE XVI. The EXPERIMENTAL Results For TEST-100-X With $\rho=1$

\begin{tabular}{|l|c|c|c|c|c|c|c|c|c|}
\hline \multirow{2}{*}{ instances } & \multicolumn{3}{|c|}{ VND } & \multicolumn{3}{c|}{ GVNS } & \multicolumn{3}{c|}{ A-GVNS } \\
\cline { 2 - 11 } & best.sol & aver.sol & time & best.sol & aver.sol & time & best.sol & aver.sol & Time \\
\hline test-100-1 & - & - & - & - & - & - & - & - & 345.1 \\
\hline test-100-2 & - & - & - & - & - & - & 39767 & 39767 & 346.7 \\
\hline test-100-3 & - & - & - & - & - & - & 37818 & 37818 & 347.8 \\
\hline test-100-4 & - & - & - & 40216 & 40216 & 341.2 & 36414 & 36414 & 346.3 \\
\hline test-100-5 & - & - & - & - & - & - & 39177 & 39177 & 349.1 \\
\hline test-100-6 & - & - & - & - & - & - & 42625 & 42625 & 347.5 \\
\hline test-100-7 & - & - & - & 44074 & 44074 & 342.2 & 37165 & 37165 & 348.4 \\
\hline test-100-8 & - & - & - & - & - & - & 41231 & 41231 & 345.2 \\
\hline test-100-9 & - & - & - & - & - & - & 40136 & 40136 & 347.3 \\
\hline test-100-10 & - & - & - & - & - & - & - & - & 348.4 \\
\hline test-100-11 & - & - & - & - & - & - & 43907 & 43907 & 348.4 \\
\hline test-100-12 & - & - & - & 40920 & 40920 & 341.3 & 36345 & 36345 & 347.9 \\
\hline test-100-13 & - & - & - & 44416 & 44416 & 340.6 & 39096 & 39096 & 346.1 \\
\hline test-100-14 & - & - & - & - & - & - & 39892 & 39892 & 349.5 \\
\hline test-100-15 & - & - & - & - & - & - & - & - & 349.6 \\
\hline test-100-16 & - & - & - & - & - & 344.2 & 39742 & 39742 & 349.8 \\
\hline test-100-17 & - & - & - & - & - & 342.2 & 43267 & 43267 & 348.7 \\
\hline test-100-18 & - & - & - & - & - & 340.1 & 41546 & 41546 & 348.4 \\
\hline test-100-19 & - & - & - & - & - & 341.6 & 39192 & 39192 & 350.0 \\
\hline test-100-20 & - & - & - & - & - & - & - & - & 346.2 \\
\hline
\end{tabular}

TABLE XVII. The EXPERIMENTAL Results For TeSt-100-X With $\rho=0.95$

\begin{tabular}{|c|c|c|c|c|c|c|c|c|c|c|c|}
\hline \multirow{2}{*}{ instances } & \multicolumn{3}{|c|}{ VND } & \multicolumn{4}{|c|}{ GVNS } & \multicolumn{4}{|c|}{ A-GVNS } \\
\hline & best.sol & aver.sol & time & best.sol & aver.sol & Gap $_{2}$ & time & best.sol & aver.sol & $\mathrm{Gap}_{2}$ & time \\
\hline test-100-1 & 39552 & 39845 & 7.53 & 37717 & 38112.36 & -4.64 & 339.2 & 37532 & 37927.09 & -5.11 & 345.4 \\
\hline test-100-2 & 52136 & 52386.91 & 6.98 & 38225 & 38770 & -26.68 & 341.2 & 38008 & 38580.64 & -27.10 & 342.5 \\
\hline test-100-3 & 51184 & 51799.36 & 7.2 & 38428 & 38747.45 & -24.92 & 340.1 & 38362 & 38632.64 & -25.05 & 342.6 \\
\hline test-100-4 & 63715 & 63986.64 & 7.75 & 38372 & 38860.45 & -39.78 & 340.3 & 38178 & 38615.91 & -40.08 & 347.8 \\
\hline test-100-5 & 43053 & 43476.64 & 6.95 & 38571 & 38815.27 & -10.41 & 339 & 38525 & 38712.09 & -10.52 & 344.4 \\
\hline test-100-6 & 50311 & 51950.18 & 7.35 & 34873 & 35530.64 & -30.69 & 342.4 & 34626 & 35194.82 & -31.18 & 343.1 \\
\hline test-100-7 & 39333 & 39792.36 & 7.29 & 36698 & 37335.18 & -6.70 & 338.1 & 36368 & 37041.82 & -7.54 & 345.2 \\
\hline test-100-8 & 51052 & 51674 & 7.53 & 39335 & 40114.91 & -22.95 & 342.3 & 39219 & 39872.09 & -23.18 & 345.3 \\
\hline test-100-9 & 39003 & 39445.45 & 7.2 & 36018 & 36508 & -7.65 & 340.6 & 35990 & 36335.55 & -7.73 & 342.2 \\
\hline test-100-10 & 49218 & 49567.55 & 7.78 & 37574 & 37974.36 & -23.66 & 338.5 & 37492 & 37811.55 & -23.82 & 345.6 \\
\hline test-100-11 & 49218 & 49567.55 & 7.72 & 37574 & 37974.36 & -23.66 & 341.4 & 37492 & 37811.55 & -23.82 & 343.9 \\
\hline test-100-12 & 50654 & 51481.55 & 7.5 & 37364 & 37574.91 & -26.24 & 339.1 & 36887 & 37466.45 & -27.18 & 343.5 \\
\hline test-100-13 & 41845 & 43332.55 & 7.26 & 39213 & 39589.27 & -6.29 & 341.7 & 39020 & 39383.73 & -6.75 & 347 \\
\hline test-100-14 & 57861 & 58539.27 & 7.09 & 40433 & 40929.73 & -30.12 & 340.2 & 40329 & 40725.73 & -30.30 & 347 \\
\hline test-100-15 & 53683 & 54339 & 7.38 & 37200 & 37472.36 & -30.70 & 339.3 & 37137 & 37346.55 & -30.82 & 342.2 \\
\hline test-100-16 & 51737 & 52904.73 & 7 & 39283 & 39627 & -24.07 & 339.3 & 39134 & 39502.36 & -24.36 & 345.9 \\
\hline test-100-17 & 54215 & 54825.82 & 7.41 & 37473 & 38204.27 & -30.88 & 341.5 & 36987 & 37820.27 & -31.78 & 342.8 \\
\hline test-100-18 & 39935 & 40116.91 & 7.52 & 36157 & 36522.73 & -9.46 & 338.6 & 36107 & 36391.45 & -9.59 & 343.5 \\
\hline test-100-19 & 53557 & 53704.36 & 7.78 & 37558 & 37915.82 & -29.87 & 339.8 & 37386 & 37680.09 & -30.19 & 342.5 \\
\hline test-100-20 & 54155 & 55492.36 & 6.96 & 36810 & 37000.55 & -32.03 & 338.6 & 36534 & 36903.36 & -32.54 & 344.6 \\
\hline Aver & & & 7.36 & & & -22.07 & 340.06 & & & -22.43 & 344.35 \\
\hline
\end{tabular}


TABLE XVIII. THE EXPERIMENTAL RESUlTS FOR TEST-100-X WITH $\rho=0.75$

\begin{tabular}{|c|c|c|c|c|c|c|c|c|c|c|c|}
\hline \multirow{2}{*}{ instances } & \multicolumn{3}{|c|}{ VND } & \multicolumn{4}{|c|}{ GVNS } & \multicolumn{4}{|c|}{ A-GVNS } \\
\hline & best.sol & aver.sol & time & best.sol & aver.sol & Gap $_{2}$ & time & best.sol & aver.sol & $\mathrm{Gap}_{2}$ & time \\
\hline test-100-1 & 39782 & 40086.18 & 7.53 & 35701 & 35835.73 & -10.26 & 339.2 & 35696 & 35778.18 & -10.27 & 345.4 \\
\hline test-100-2 & 40850 & 41751 & 6.98 & 36254 & 36380.64 & -11.25 & 341.2 & 36178 & 36326.64 & -11.44 & 342.5 \\
\hline test-100-3 & 49744 & 50145.18 & 7.2 & 36966 & 37039.36 & -25.69 & 340.1 & 36168 & 36921.45 & -27.29 & 342.6 \\
\hline test-100-4 & 37826 & 38207.36 & 7.75 & 36481 & 36806.55 & -3.56 & 340.3 & 36466 & 36713 & -3.60 & 347.8 \\
\hline test-100-5 & 55970 & 57384.36 & 6.95 & 37052 & 37185.73 & -33.80 & 339 & 36930 & 37119.64 & -34.02 & 344.4 \\
\hline test-100-6 & 66094 & 67482.09 & 7.35 & 34806 & 34950.82 & -47.34 & 342.4 & 34677 & 34900.18 & -47.53 & 343.1 \\
\hline test-100-7 & 51980 & 52418 & 7.29 & 35900 & 36041.09 & -30.93 & 338.1 & 35711 & 35954.91 & -31.30 & 345.2 \\
\hline test-1 & 47856 & 48351.45 & 7.53 & 36787 & 36942.82 & -23.13 & 342.3 & 36645 & 36869 & -23.43 & 345.3 \\
\hline test-100-9 & 56256 & 57560 & 7.2 & 34731 & 34890.91 & -38.26 & 340.6 & 34713 & 34827.64 & -38.29 & 342.2 \\
\hline test-100-10 & 39514 & 39720.09 & 7.78 & 36031 & 36235.36 & -8.81 & 338.5 & 35849 & 36143.27 & -9.28 & 345.6 \\
\hline test-100-11 & 39514 & 39720.09 & 7.72 & 36031 & 36235.36 & -8.81 & 341.4 & 35849 & 36143.27 & -9.28 & 343.9 \\
\hline test-100-12 & 36985 & 37368.64 & 7.5 & 35015 & 35428.82 & -5.33 & 339.1 & 34983 & 35313.36 & -5.41 & 343.5 \\
\hline test & 40200 & 40584.73 & 7.26 & 37546 & 37609.82 & -6.60 & 341.7 & 37422 & 37570.64 & -6.91 & 347 \\
\hline test-1 & 40118 & 40654.27 & 7.09 & 37626 & 37681.64 & -6.21 & 340.2 & 37588 & 37657.91 & -6.31 & 347 \\
\hline test-100-15 & 39845 & 40294.36 & 7.38 & 36564 & 36705.09 & -8.23 & 339.3 & 36400 & 36642 & -8.65 & 342.2 \\
\hline test-100-16 & 46970 & 47708.45 & $\overline{7}$ & 35927 & 36200.73 & -23.51 & 339.3 & 35866 & 36104.91 & -23.64 & 345.9 \\
\hline test-100-17 & 60588 & 60978.18 & 7.41 & 36142 & 36294.55 & -40.35 & 341.5 & 36114 & 36239.18 & -40.39 & 342.8 \\
\hline test-100-18 & 38746 & 39396.64 & 7.52 & 35692 & 35763.64 & -7.88 & 338.6 & 35647 & 35732.45 & -8.00 & 343.5 \\
\hline test-100-19 & 52204 & 52881.45 & 7.78 & 36304 & 36678.27 & -30.46 & 339.8 & 36158 & 36544.64 & -30.74 & 342.5 \\
\hline test-100-20 & 38485 & 38936.91 & 6.96 & 35364 & 35583.91 & -8.11 & 338.6 & 35351 & 35508.09 & -8.14 & 344.6 \\
\hline Aver & & & 7.36 & & & -18.93 & 340.06 & & & -19.20 & 344.35 \\
\hline
\end{tabular}

TABLE XIX. The EXPERIMENTAL RESUlts For TeSt-100-X With $\rho=0.5$

\begin{tabular}{|c|c|c|c|c|c|c|c|c|c|c|c|}
\hline \multirow{2}{*}{ instances } & \multicolumn{3}{|c|}{ VND } & \multicolumn{4}{|c|}{ GVNS } & \multicolumn{4}{|c|}{ A-GVNS } \\
\hline & best.sol & aver.sol & time & best.sol & aver.sol & Gap2 $_{2}$ & time & best.sol & aver.sol & Gap2 $_{2}$ & time \\
\hline test-100-1 & 58004 & 58231.27 & 7.09 & 36013 & 36341.27 & -37.91 & 336 & 35915 & 36216.82 & -38.08 & 340.8 \\
\hline test-100-2 & 38230 & 39685.36 & 7.56 & 36423 & 36873.91 & -4.73 & 337 & 36279 & 36727.18 & -5.10 & 341.2 \\
\hline test-100-3 & 43252 & \begin{tabular}{|l|l}
44277.82 \\
\end{tabular} & 7.29 & 36307 & 36463.36 & -16.06 & 339.4 & 36292 & 36399.27 & -16.09 & 340.6 \\
\hline test-100-4 & 38100 & 38574.55 & 7.38 & 35931 & 36153.18 & -5.69 & 336.4 & 35882 & 36075.27 & -5.82 & 342.5 \\
\hline test-100-5 & 8561 & \begin{tabular}{|l}
39019.45 \\
\end{tabular} & 6.85 & 36999 & 37210.91 & -4.05 & 336.1 & 36988 & 37147.36 & -4.08 & 345.4 \\
\hline test-100-6 & 64673 & 65026.91 & 7.27 & 34494 & 34570.27 & -46.66 & 339.7 & 34428 & 34534.36 & -46.77 & 345.7 \\
\hline test-100-7 & 38083 & 38466.27 & 6.8 & 35987 & 36059.82 & -5.50 & 338.3 & 35946 & 36022.91 & -5.61 & 344.8 \\
\hline test-100-8 & 58805 & 59305.45 & 7.57 & 36448 & 36658.09 & -38.02 & 336.8 & 36322 & 36575.45 & -38.23 & 344.8 \\
\hline test-1 & 53032 & 5356 & 6.98 & 34716 & 34806.27 & -34.54 & 339.1 & 34704 & 34771.27 & -34.56 & 344.2 \\
\hline test-100-10 & 58146 & \begin{tabular}{|l}
58615.55 \\
\end{tabular} & 7.45 & 35942 & 36054.55 & -38.19 & 338.2 & 35934 & 36019.18 & -38.20 & 343.7 \\
\hline test-100-11 & 58146 & 58615.55 & 6.6 & 35942 & 36054.55 & -38.19 & 340.7 & 35934 & 36019.18 & -38.20 & 344.5 \\
\hline test-100-12 & 49400 & \begin{tabular}{|l|}
50094.55 \\
\end{tabular} & 7.53 & 35354 & 35540.09 & -28.43 & 336.4 & 35235 & 35450.36 & -28.67 & 342.4 \\
\hline test-100-13 & 62370 & \begin{tabular}{|l}
62851.27 \\
\end{tabular} & 7.37 & 37268 & 37371.18 & -40.25 & 338.6 & 37075 & 37284.73 & -40.56 & 344.2 \\
\hline test-100-14 & 41140 & \begin{tabular}{|l}
42124.82 \\
\end{tabular} & 7.12 & 37325 & 37543.27 & -9.27 & 336.1 & 37088 & 37457.45 & -9.85 & 340.9 \\
\hline test-100-15 & 55569 & 5712 & 7.23 & 36220 & 36407.36 & -34.82 & 339.9 & 36072 & 36333.45 & -35.09 & 343.3 \\
\hline test-100-16 & 42710 & 43159.55 & 6.98 & 36000 & 36169.01 & -15.71 & 339.8 & 35945 & 36103.09 & -15.84 & 345.5 \\
\hline test-100-17 & 42307 & \begin{tabular}{|l}
43157.18 \\
\end{tabular} & 6.99 & 36372 & 36436.11 & -14.03 & 339.4 & 36287 & 36393.18 & -14.23 & 340.5 \\
\hline test-100-18 & 51547 & \begin{tabular}{|l|}
52389.09 \\
\end{tabular} & 6.71 & 35742 & 35879.45 & -30.66 & 338.9 & 35718 & 35828.45 & -30.71 & 341.5 \\
\hline test-100-19 & 49862 & 50832.36 & 7.57 & 36196 & 36382.64 & -27.41 & 339.5 & 36084 & 36296.73 & -27.63 & 345.8 \\
\hline test-100-20 & 55788 & 56354.27 & 6.66 & 35434 & 35627.14 & -36.48 & 337.5 & 35299 & 35541.09 & -36.73 & 340.8 \\
\hline Aver & & & 7.15 & & & -25.33 & 338.19 & & & -25.50 & 343.16 \\
\hline
\end{tabular}


TABLE XX. THE EXPERIMENTAL RESUlTS FOR TEST-100-X WITH $\rho=0$

\begin{tabular}{|c|c|c|c|c|c|c|c|c|c|c|c|}
\hline \multirow{2}{*}{ instances } & \multicolumn{3}{|c|}{ VND } & \multicolumn{4}{|c|}{ GVNS } & \multicolumn{4}{|c|}{ A-GVNS } \\
\hline & est.sol & aver.sol & time & est.sol & aver.sol & $\mathrm{Gap}_{2}$ & time & best.sol & aver.sol & Gap $2_{2}$ & time \\
\hline test-100-1 & 40252 & 41601.7 & 7.11 & 36082 & 36239.18 & -10.36 & 337.6 & 36055 & 361817 & -10.43 & 341.5 \\
\hline test- $100-2$ & 5017 & 55796.8 & 7.28 & 5536 & 36635.27 & -20.60 & 337.7 & 36497 & 36561.9 & -20.69 & 340.4 \\
\hline test-100-3 & 7134 & 66605.8 & 6.89 & 36027 & 36198.55 & -23.56 & 335.3 & 35990 & 36097.9 & -23.64 & 342.5 \\
\hline test- $100-4$ & 43528 & 48294.5 & 6.62 & 36221 & 36597.45 & -16.79 & 337.1 & 36005 & 36409.2 & -17.28 & 338.8 \\
\hline test-1 & 202 & & 6.59 & (5) & 82 & -19.85 & 3. & 30 & 7.9 & -19.85 & 343.1 \\
\hline test- & 522 & & 6.42 & 1866 & 350 & -23.41 & 334.9 & 50 & 57.6 & -23.43 & 342.6 \\
\hline test-10 & 590 & 39915.6 & 6.8 & 35618 & 357 & -10.03 & 339.2 & 5608 & 95.4 & -10.06 & 343.4 \\
\hline test-1 & 45330 & 59064.5 & 6.98 & 37013 & 37219.36 & -18.35 & 338.4 & 36996 & 37119.5 & -18.39 & 339.4 \\
\hline test-100-9 & 42800 & 57751.5 & 6.54 & 34712 & 35015.55 & -18.90 & 339.5 & 34649 & 34867.4 & -19.04 & 343.5 \\
\hline test & 831 & & 6.71 & 35889 & & -23.36 & 336.4 & 35825 & 52.3 & -23.50 & 338.9 \\
\hline test-1 & 6831 & 555 & 6.58 & 35889 & 36046.09 & -23.36 & 337.6 & 35825 & 35952.3 & -23.50 & 343.9 \\
\hline test-1 & 36743 & 36942.5 & 6.77 & 34987 & 35247.36 & -4.78 & 336.2 & 34947 & 35130.2 & -4.89 & 339.1 \\
\hline test-1 & 37999 & 38647.8 & 6.71 & 36978 & 37245.73 & -2.69 & 338.3 & 36942 & 37129.6 & -2.78 & 342.1 \\
\hline test-100-14 & 39466 & 40796 & 6.6 & 36819 & 37283.18 & -6.71 & 334.2 & 36774 & 37100.3 & -6.82 & 338.9 \\
\hline test-1 & 42777 & 52823.5 & 7.29 & 36138 & 363 & -15.52 & 337.3 & 36111 & 36262.2 & -15.58 & 341.7 \\
\hline test- & 38495 & & 7.01 & 35577 & 357 & -7.58 & 339.7 & 35532 & 29.8 & -7.70 & 338.8 \\
\hline & 2166 & & 6.51 & 36337 & 364 & -13.82 & 336.5 & 36333 & 36421 & -13.83 & 340.6 \\
\hline test- & 39192 & 408 & 6.95 & 35484 & 35647.36 & -9.46 & 338.9 & 35372 & 35537 & -9.75 & 343 \\
\hline test-100-19 & 39607 & 40387.8 & 6.53 & 36031 & 36245.64 & -9.03 & 337.3 & 35901 & 36149 & -9.36 & 340.6 \\
\hline test- $100-20$ & 38396 & 39399.2 & 7.15 & 35535 & 35669.91 & -7.45 & 338.8 & 35534 & 35615.9 & -7.45 & 340.3 \\
\hline Aver & & & 6.80 & & & -14.28 & 337.46 & & & -14.40 & 341.16 \\
\hline
\end{tabular}

TABLE XXI. TheAverageGap 2

\begin{tabular}{|c|c|c|c|c|c|c|c|c|c|c|}
\hline \multirow{3}{*}{ instances } & \multicolumn{2}{|c}{$\rho=0$} & \multicolumn{2}{c|}{$\rho=0.5$} & \multicolumn{2}{c|}{$\rho=0.75$} & \multicolumn{2}{c|}{$\rho=0.95$} & \multicolumn{2}{c|}{$\rho=1$} \\
\cline { 2 - 11 } & GVNS & $\begin{array}{c}\text { A- } \\
\text { GVNS }\end{array}$ & GVNS & $\begin{array}{c}\text { A- } \\
\text { GVNS }\end{array}$ & GVNS & $\begin{array}{c}\text { A- } \\
\text { GVNS }\end{array}$ & GVNS & $\begin{array}{c}\text { A- } \\
\text { GVNS }\end{array}$ & GVNS & $\begin{array}{c}\text { A- } \\
\text { GVNS }\end{array}$ \\
\hline 30 & -6.19 & -6.27 & -9.59 & -9.96 & -9.90 & -10.09 & -5.06 & -6.38 & - & - \\
\hline 40 & -9.35 & -9.35 & -11.74 & -11.99 & -15.01 & -15.19 & -2.43 & -3.47 & - & - \\
\hline 50 & -9.45 & -9.63 & -18.43 & -18.66 & -16.66 & -16.87 & -6.31 & -7.15 & - & - \\
\hline 100 & -14.28 & -14.40 & -25.33 & -25.50 & -18.93 & -19.20 & -22.07 & -22.43 & - & - \\
\hline
\end{tabular}

Since $\rho=1$, there is no the Gap2 value because the VNS does not provide any feasible sol utions in these cases.

TABlE XXII. The EXPERImental Results For A. SALEhipour et Al.'s Dataset

\begin{tabular}{|c|c|c|c|c|c|r|}
\hline \multirow{3}{*}{ Instances } & \multicolumn{2}{|c|}{ GRASP-VNS } & \multicolumn{2}{|c|}{ ILS } & \multicolumn{2}{c|}{ A-GVNS } \\
\cline { 2 - 7 } & $\begin{array}{c}\text { gap } \\
\text { ] } \%\end{array}$ & $T$ & $\begin{array}{l}\text { gap } \\
] \%[\end{array}$ & cTime & $\begin{array}{c}\text { gap } \\
] \%[\end{array}$ & cTime \\
\hline 10 & 33.04 & 0.00 & 33.04 & 0.00 & $\mathbf{3 3 . 0 4}$ & 0.00 \\
\hline 20 & 40.34 & 0.04 & 39.29 & 0.05 & $\mathbf{3 9 . 2 9}$ & 0.18 \\
\hline 50 & 47.20 & 3.54 & 43.97 & 1.36 & $\mathbf{4 3 . 9 7}$ & 9.38 \\
\hline 100 & 44.28 & 103.92 & 40.82 & 18.94 & $\mathbf{4 0 . 8 2}$ & 25.82 \\
\hline 200 & 38.77 & 3995.0 & 38.14 & 178.72 & $\mathbf{3 8 . 1 4}$ & 198.2 \\
\hline
\end{tabular}

TABLE XXIII. THE EXPERIMENTAL RESUlts FOR THE INSTANCES IN TSPLIB

\begin{tabular}{|l|r|r|r|c|}
\hline Instances & \multicolumn{1}{c|}{ OPT } & best.sol & aver.sol & $T$ \\
\hline dantzig42 & 12528 & $\mathbf{1 2 5 2 8}$ & 12528 & 5.16 \\
\hline att48 & 209320 & $\mathbf{2 0 9 3 2 0}$ & 209320 & 12.4 \\
\hline eil51 & 10178 & $\mathbf{1 0 1 7 8}$ & 10178 & 17.7 \\
\hline berlin52 & 143721 & $\mathbf{1 4 3 7 2 1}$ & 143721 & 17.2 \\
\hline st70 & 20557 & $\mathbf{2 0 5 5 7}$ & 20557 & 41.2 \\
\hline KroA100 & 983128 & $\mathbf{9 8 3 1 2 8}$ & 983128 & 67.5 \\
\hline KroB100 & 983128 & $\mathbf{9 8 3 1 2 8}$ & 983128 & 64.4 \\
\hline KroC100 & 961324 & $\mathbf{9 6 1 3 2 4}$ & 961324 & 64.4 \\
\hline KroD100 & 976965 & $\mathbf{9 7 6 9 6 5}$ & 976965 & 62.3 \\
\hline
\end{tabular}

